\title{
1 Convergent evolution of mevalonate pathway in 2 Inonotus obliquus and Betula pendula.
}

Omid Safronov ${ }^{1,2,3}$, Guleycan Lutfullahoglu Bal ${ }^{2}$, Nina Sipari ${ }^{4}$, Maya Wilkens ${ }^{5}$, Pezhman Safdari ${ }^{1}$, Olli-Pekka Smolander ${ }^{6}$, Jenna Lihavainen ${ }^{7}$, Niko Silvan ${ }^{8}$, Sitaram Rajaraman ${ }^{1}$, Pia K. Laine ${ }^{2}$, Lars G Paulin $^{2}$, Petri Auvinen ${ }^{2}$, Tytti Sarjala ${ }^{8}$, Kirk Overmyer ${ }^{1}$, Jaakko Kangasjärvi ${ }^{1}$, Brendan Battersby ${ }^{2,3}$, Uwe Richter ${ }^{2,9, *}$, Jarkko Salojärvi ${ }^{1,10, *}$.

${ }^{1}$ Organismal and Evolutionary Biology Research Program, Faculty of Biological and

Environmental Sciences, and Viikki Plant Science Centre, University of Helsinki,

Finland.

${ }^{2}$ Institute of Biotechnology, HiLIFE, University of Helsinki, Helsinki, Finland.

${ }^{3}$ Molecular and Integrative Biosciences Research Program, Faculty of Biological and

Environmental Sciences, and Viikki Plant Science Centre, University of Helsinki,

Finland.

${ }^{4}$ Viikki Metabolomics Unit, Faculty of Biological and Environmental Sciences, University of Helsinki, Helsinki Finland.

${ }^{5}$ Current address: Quantitative Proteomics, Institute of Molecular Biology, Mainz, Germany.

${ }^{6}$ Department of Chemistry and Biotechnology, Tallinn University of Technology, Tallinn, Estonia.

${ }^{7}$ Department of Plant Physiology, Umeå Plant Science Centre, Umeå University, Umeå, Sweden.

${ }^{8}$ Biomass Characterization and Properties Group, Production Systems Unit, Natural Resources Institute Finland, Helsinki, Finland.

${ }^{9}$ Wellcome Centre for Mitochondrial Research, Biosciences Institute, Newcastle University, UK.

${ }^{10}$ School of Biological Sciences, Nanyang Technological University, Singapore, Singapore.

*Corresponding authors: uwe.richter@helsinki.fi,jarkko@ntu.edu.sg

\section{Abstract}

Inonotus obliquus, Chaga mushroom, is a fungal species from Hymenochaetaceae family (Basidiomycota) which has been widely used for traditional medicine in Europe and Asia. Here, chaga genome was sequenced using Pacbio sequencing into a 50.7Mbp assembly consisting of 301 primary contigs with an N50 value of $375 \mathrm{kbp}$. Genome evolution analyses revealed a lineagespecific whole genome duplication event and an expansion of Cytochrome P450 superfamily. Fungal biosynthetic clusters were enriched for tandemly duplicated genes, suggesting that biosynthetic pathway evolution has proceeded through small-scale duplications. Metabolomic fingerprinting 
confirmed a highly complex terpene biosynthesis chemistry when compared against related fungal species lacking the genome duplication event.

\section{Introduction}

Inonotus obliquus, Chaga mushroom, is a fungal species from Hymenochaetaceae family (Basidiomycota) distributed across the boreal forest zone in the Northern hemisphere. It causes aggressive white rot disease mainly among Betula family members (Blanchette, 1982), but upon suitable conditions it can infect also other tree species such as oaks, poplars, ashes and maples (Ryvarden \& Gilbertson, 1993). White rot disease is the result of lignin degradation (having darker color) while the light-coloured cellulose is left intact. The infection starts when I. obliquus spores get access to the hardwood of the stem through an opening or wounded bark. At the later stages of the infection, I. obliquus appears as a sterile conk, a solid charcoal-black mass on the surface of bark (Blanchette, 1982). The sterile conk has been used in traditional medicine in many cultures. A large body of research on biochemical compounds extracted from the conk suggests that the species may have a wide range of pharmaceutical, medicinal, and industrial applications (Ma, Chen, Dong, \& Lu, 2013; Nagajyothi, Sreekanth, Lee, \& Lee, 2014; Song, Liu, Kong, Chang, \& Song, 2013; Yan et al., 2014).

Betulin (BE) and betulinic acid are highly abundant triterpenoids in the bark of all birch family members involved in protection against fungi, bacteria and viruses; they collectively form $30-60 \%$ of total tissue composition, depending on the species and the tissue type (Holonec, Ranga, Crainic, Truța, \& Socaciu, 2012; P. Kovalenko et al., 2009; Safronov et al., 2019). Both betulinate compounds are being studied for industrial applications (Šiman et al., 2016), and as therapeutic substances in oncology (Król, Kiełbus, Rivero-Müller, \& Stepulak, 2015) and infectious diseases (fungal, bacterial, and viral infections) (Gong et al., 2004; Salin et al., 2010; Shai, McGaw, Aderogba, Mdee, \& Eloff, 2008). In plants, the biosynthesis of betulinate compounds starts with squalene, a product of mevalonate pathway, and involves two enzymatic steps where squalene is first converted to lupeol via lupeol synthase and then to betulinate by lupeol monooxygenase, an enzyme which is a member of the large family of cytochrome P450 monooxygenases, more specifically subfamily 716 (CYP716). Betulin biosynthesis is found across a wide taxonomic range in plants, from Malvales $(\mathrm{H}$. J. Zhang et al., 2003), Fagales (Safronov et al., 2019), Rosales (Andre et al., 2013; S. Zhao et al., 2015), Fabales (Wu, Niu, Bakur, Li, \& Chen, 2017), Vitales (Fukushima et al., 2011), and Asterales (Siddiqui et al., 2019) to Arecales (Khelil, Jardé, Cabello-Hurtado, Ould-el-Hadj Khelil, \& Esnault, 2016; Koolen et al., 2012), suggesting either ancestral origin or convergent evolution. A comparative genomic analysis of the bark tissue in silver birch (Betula pendula) and grey alder (Alnus glutinosa) revealed birch- 
specific evolution of mevalonate pathway (MVA), where a tandem duplication of lupeol synthase colocalized with lupeol 28-monooxygenase was suggested as the reason for increased production of betulinate compounds in birch phellem (Safronov et al., 2019). Interestingly, in addition to plants, betulinate compounds have also been identified in diverse range of fungal species from Eurotiales (Khouloud Barakat, 2016), Hymenochaetales (Yin, Cui, \& Ding, 2008), and Polyporales (Alresly et al., 2015) families, even though no members of CYP716 gene family have yet been identified or characterized in fungi. Birch fungal pathogens Inonotus obliquus and Fomitopsis betulina are such examples of fungal species that produce BE and BA compounds (Alresly et al., 2015; Yin et al., 2008), even though the compounds are anti-fungal by nature. The evolution of betulinate biosynthesis in these two fungal species is not known, but one can hypothesize it to be the result of either convergent evolution or horizontal gene transfer (HGT) of the responsible cytochrome P450 monooxygenase enzymes from the host species. There exists a recent study on the diversification and distribution of CYP716 enzyme in eudicots (Miettinen et al., 2017), but no studies of this enzyme in fungal species have been carried out, and the enzymes underlying betulin production in the fungal species, known to produce betulinate compounds, have not yet been identified.

The CYP450 monooxygenase enzymes are among the oldest and largest gene families, encompassing both prokaryotic and eukaryotic organisms (Sezutsu, Le Goff, \& Feyereisen, 2013). They act as key enzymes for detoxification of toxic compounds, and they have an important function in secondary metabolism related to adaptation to environmental conditions. The low sequence similarity, high functional diversity and enzymatic promiscuity among CYP450 monooxygenase enzymes makes functional predictions difficult. The CYP450s are generally classified into families and subfamilies based on sequence similarity; the sequences with identity $>40 \%$ are assigned into families and sequences with $>55 \%$ similarity into their own subfamilies; novel candidates with lower identity to the set of identified CYP450s form new candidate families. Based on these criteria, so far over 800 different CYP families have been identified (Lepesheva et al., 2008).

In this study we sequenced and assembled an I. obliquus genome from an isolate from Merikarvia region in Finland. The genome was annotated using $a b$ initio gene model prediction and spliced transcript data obtained from total RNA sequencing. We carried out comparative genomic and gene family expansion analysis among 16 Basidiomycete and 3 Ascomycetes species together with QTOF/MS) in five strains of I. obliquus and one Fomitiporia mediterranea strain, focusing on the quantification of betulin (BE) and betulinic acid (BA) abundances across the samples. To confirm our functional predictions we cloned the candidate lupeol synthase from B. pendula and CYP450 
100

101

102

103

104

105

106

107

108

109

110

111

112

113

monooxygenase enzymes from both $B$. pendula and $I$. obliquus and tested their ability to produce betulin compounds.

\section{Materials and methods}

\section{Sample collection}

Four Inonotus obliquus strains (Supp. table 1) were collected and isolated from different regions in Finland and one from Altai mountains in Russia. The strain from Merikarvia was selected for whole

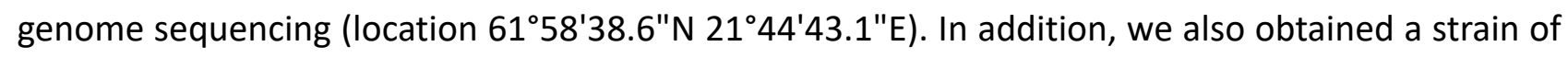
Fomitiporia mediterranea as an outgroup to chaga (Mycobank: MB384943). All samples were cultivated on Hagem agar overlayed by a cellophane membrane.

The isolation of chaga mushrooms from the host trees was done by cutting a piece of the conk (Supp. Fig 1), which was then laid on agar plate after short $\mathrm{H}_{2} \mathrm{O}_{2}$ bath. The samples were re-cultured repeatedly and sequenced for internal transcribed spacer 1 (ITS1) [TCCGTAGGTGAACCTGCGG] and ITS4 [TCCTCCGCTTATTGATATGC] regions confirm the species assignment of $I$. obliquus isolate.

\section{RNA isolation, sequencing, and de novo assembly of transcriptome}

To isolate the total RNA from I. obliquus, the method from Chang et al. (Chang, Puryear, \& Cairney, 1993) was used. Briefly, the I. obliquus was inoculated and grown on autoclaved wood dust from a clone of $B$. pendula (12 years old tree, $167 \mathrm{~cm}^{2}$ disk, dry weight of 200 grams) sequenced for $B$. pendula reference genome (Salojärvi et al., 2017). A total of 150 milligrams of ground sample (mortar and pestle, and liquid $\mathrm{N}_{2}$ ) was transferred on ice for 30 seconds, and $500 \mu \mathrm{l}$ of pre-warmed $\left(+65-68^{\circ} \mathrm{C}\right)$ extraction buffer (2\% CTAB, 2\% PVP K-30, $100 \mathrm{mM}$ Tris- $\mathrm{HCl}$ [pH 8.0], $25 \mathrm{mM}$ EDTA, $2 \mathrm{M}$ $\mathrm{NaCl}$, and $200 \mu \mathrm{l} \beta-\mathrm{MeOH} / 10 \mathrm{ml}$ of extraction buffer) was added and vortexed vigorously. Extraction was carried out three times with chloroform:isoamyl alcohol (24:1) by spinning at 200-300 rpm for 15 minutes, and then centrifuging at $10000 \mathrm{rpm} 15$ minutes. Then, $1 / 4$ volume $10 \mathrm{M} \mathrm{LiCl}$ was added and left to precipitate on ice overnight. The overnight sample was centrifuged with $10000 \mathrm{rpm}$ for 20-30 minutes at $+4^{\circ} \mathrm{C}$, and the resulting pellet was dissolved in $500 \mu \mathrm{l}$ of pre-warmed $\left(+65^{\circ} \mathrm{C}\right)$ sodium dodecyl sulfate-Tris-HCl-EDTA (SSTE) buffer, and extracted once (or several times, if necessary) with chloroform:isoamyl alcohol (24:1). The mixture was precipitated by adding 2 volumes of absolute EtOH (place at $-20^{\circ} \mathrm{C}$ overnight), and centrifuged at $13000 \mathrm{rpm}$, for 20-30 minutes at $+4^{\circ} \mathrm{C}$. the precipitate was washed with $70 \% \mathrm{EtOH}$, after which the pellet was dried, and then dissolved in 10-30 $\mu$ l RNase-free water, and RNase inhibitor was added.

TruSeq stranded mRNA kit was used to construct the RNA-seq library. The cDNA was synthesized from $5 \mu$ l of total RNA extracted from reference I. obliquus plate using random hexamers. DNA polymerase I and dUTP nucleotides were used to synthesize the second strand of 
133 cDNA. Then, double stranded cDNA were purified, and ends were repaired. Library preparation 134 was continued by A-tailing, and ligation of $Y$-adaptors containing indexes from the kit. The 135 fragments were amplified using polymerase chain reaction (PCR), followed by purification steps 136 using AMPure XP. The sequencing was carried out in HiScan SQ platform (paired-end 88 bp +74 137 bp).

138 The raw paired end RNA-seq data were controlled for quality using FastQC v0.11.2 (Andrews). 139 Trimmomatic v0.33 (Bolger, Lohse, \& Usadel, 2014) was used in pair-end mode to remove the 140 adapters, barcodes, low quality bases from both ends of each sequence, and reads shorter than 25 141 base pairs (LEADING:20, TRAILING:20, MINLEN:25, -phred33). After the removal of duplicate 142 sequences, the unpaired sequences were mapped to $I$. obliquus reference genome using Tophat2 143 (Kim et al., 2013) for junction discoveries (-i:10, and --coverage-search); paired end reads were 144 mapped separately (Tophat2; -i:10, and --coverage-search). The aligned reads were separated 145 according to their orientation on reference genome to forward and reverse strands, which were then 146 aligned individually by Trinity v2.1.1, using --genome_guided_bam, and -147 genome_guided_max_intron: 1000 options (Grabherr et al., 2011) for de novo transcriptome 148 assemblies. The forward and reverse de novo transcriptome assemblies were combined, and 149 duplicated assemblies were removed using GenomeTools v1.5.1, using sequniq option (Gremme, 150 Steinbiss, \& Kurtz, 2013). The unique de novo transcriptome assemblies were clustered by using CD151 HIT v4.6 (Godzik \& Li, 2006) and aligned to I. obliquus reference genome by Program to Assemble 152 Spliced Alignments (PASA v2.2.0) (Brian J. Haas et al., 2008).

153 The processing of the publicly available RNAseq data (Fradj et al., 2019) was carried out in a 154 similar manner. Both data sets were mapped to $I$. obliquus gene models using kallisto quant v0.44.0 155 (Bray, Pimentel, Melsted, \& Pachter, 2016). The orphan reads and pair-end reads (separated during 156 preprocessing by trimmomatic) were mapped separately by using kallisto quant single (options: -157 single, -I 200, -s 20, -b 4000) and pair-end (option: -b 4000) modes, respectively. The raw count table 158 from Kallisto was imported to $\mathrm{R}$ (for both single and pair-end count tables) using tximport package 159 v1.18.0 with default options (Soneson, Love, \& Robinson, 2015). The single and pair-end counts were 160 summed together to form a single count table for each data set. Differential gene expression analysis 161 was conducted using DESeq2 (Love, Huber, \& Anders, 2014). The final tables for differentially 162 expressed genes (DEg) were filtered based on the false discovery rate adjusted $p$-value threshold of 1630.05 (p-adj. $\leq 0.05)$. 
bioRxiv preprint doi: https://doi.org/10.1101/2021.11.28.470225; this version posted November 28,2021 . The copyright holder for this preprint (which was not certified by peer review) is the author/funder, who has granted bioRxiv a license to display the preprint in perpetuity. It is made available under aCC-BY-NC-ND 4.0 International license.

\section{DNA isolation, genome assembly and annotation}

Modified version of Lodhi et al. (Lodhi, Ye, Weeden, \& I. Reisch, 1994) was used for DNA extraction from I. obliquus strains. Maximum $0.5 \mathrm{~g}$ of material was ground in liquid $\mathrm{N}_{2}$. The ground sample was transferred into ice cold Sodium chloride-Tris-EDTA (STE) buffer (1,4 M NaCl, $0 \mathrm{mM}$

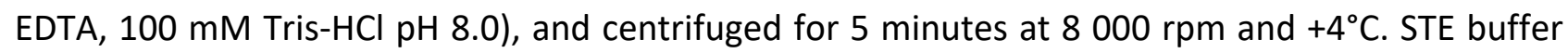
was discarded, and $10 \mathrm{ml}$ of pre-warmed $\left(60^{\circ} \mathrm{C}\right)$ cetyltrimethyl ammonium bromide (CTAB) buffer (1 liter CTAB: 20 mM EDTA, 100 mM Tris-HCl pH 8.0, 1.4 M NaCl, 2.0\% CTAB, 1.0\% PVP 40, and 2\% $\beta-\mathrm{MeOH}[50 \mu l])$ was added to pellet. Subsequently, the mixture was vortexed and incubated for 30$60 \mathrm{~min}$ at $60^{\circ} \mathrm{C}$ and cooled to the room temperature. Chloroform: isoamyl alcohol (IAA) (24:1 ration) mixture was added for extraction (centrifugation: 15 minutes, $10000 \mathrm{rpm}$ at room temperature). The supernatant was collected to a new tube and mixed with $2 X$ CTAB buffer, which was then vortexed and incubated for $30-60 \mathrm{~min}$ at $60^{\circ} \mathrm{C}$. The chloroform:IAA extraction step was repeated 23 times, followed by adding of $2 \mathrm{X}$ volume of cold $\left(-20^{\circ} \mathrm{C}\right)$ absolute ethanol $(\mathrm{EtOH})$ to supernatant. The EtOH mixture was stored for overnight at $+4^{\circ} \mathrm{C}$. The mixture then was centrifuged for 15 minutes, at $10000 \mathrm{rpm}$ and $4^{\circ} \mathrm{C}$. DNA pellet was washed with absolute EtOH $\left(-20^{\circ} \mathrm{C}\right)$ and air dried. The sample was treated for the RNA (RNase A), followed by chloroform:IAA extraction, EtOH precipitation, air drying of the DNA pellet, dissolving in DNase/RNase free water, and storing at $80^{\circ} \mathrm{C}$.

The genome of the Inonotus obliquus was sequenced with Pacific Biosciences PacBio RSII instrument using P6-C4 chemistry. Eight SMRTcells were used for sequencing the sample with movie time of 240 minutes. The number of obtained sequences was 712,759 which totaled up to $4.82 \mathrm{~Gb}$ of data with read length N50 of 9200 bp. At first, hierarchical Genome Assembly Process (HGAP) V3 implemented in SMRT Analysis package (v2.3.0) was used to generate an initial de novo genome assembly with default parameters. Mitochondrial genome contig was separated from the chromosomal contigs and circularized manually using GAP4 program (Bonfield, Smith, \& Staden, 1995). Obtained mitochondrial sequence in length of $118085 \mathrm{bp}$ and $>4000 \mathrm{X}$ sequencing coverage was polished using SMRT Analysis RS Resequencing protocol with Quiver consensus algorithm. Second, the FALCON assembly program (Chin et al., 2016) was used to generate the final de novo genome assembly with seed read length of $10000 \mathrm{bp}$. Obtained contig sequences were polished using SMRT Analysis RS Resequencing protocol with Quiver consensus algorithm with approximately $75 x$ coverage. To quantify completeness of the genome, BUSCO (v3.0, Fungi datasets, -m geno, long) (Waterhouse et al., 2017) was used. 
Repeat analysis of the contigs was carried out according to the guidelines of RepeatModeler and RepeatMasker (http://www.repeatmasker.org/, v 4.0.7). To predict the gene models, multiple evidence tracks from different platforms were obtained: $a b$ initio gene predictors based on Hidden Markov Models (HMMs), spliced transcript evidence from RNA-seq, and orthologous proteins from closely related fungal species. HMM-based models such as AUGUSTUS (v3.3.2) (Stanke \& Morgenstern, 2005), and GeneMark-ES (version 4.33; --fungus mode, and -evidence: de novo

transcriptome assembly) (Besemer \& Borodovsky, 2005) were used for $a b$ initio gene predictions. In addition, BRAKER2 (Hoff, Lange, Lomsadze, Borodovsky, \& Stanke, 2015) (options: --fungus, -rounds $=100$, and --bam) was run for $a b$ initio gene predictions. To identify the open reading frames (ORFs) within the genome, getorf (EMBOSS v6.6.0.0) program (Rice, Longden, \& Bleasby, 2000) was used (-find:1, and -maxsize: 5000). The ORFs were then queried against NR database by DIAMOND (v0.9.24, blastp, --more-sensitive) (Buchfink, Xie, \& Huson, 2014) and filtered for similarity

(sequence identity $\geq 75$, and score $\geq 300$ ); the homologous sequences above the threshold were collected. Selected ORFs were used as the input for exonerate (v2.46.2, --model:protein2genome, -minintron:10, --maxintron:1000; --percent:65) (Slater \& Birney, 2005) to map the candidate ORFs to $I$. obliquus reference genome. Additionally, orthologous proteins from 13 fungal species (Coprinopsis cinerea, Fomitiporia mediterranea, Heterobasidion annosum, Laccaria bicolor, Onnia scaura, Phanerochaete chrysosporium, Phellinus ferrugineofuscus, Porodaedalea niemelaei, Postia placenta, Puccinia graminis, Rickenella mellea, Schizopora paradoxa, Trichaptum abietinum) were aligned against $I$. obliquus reference genome with exonerate (v2.46.2, --model:protein2genome, -minintron:10, --maxintron:1000; --percent:65) (Slater \& Birney, 2005). In addition to orthologous proteins, the protein sequences discovered from BUSCO predictions were collected and aligned to reference genome by exonerate as well using the same parameters as given above (Slater \& Birney, 2005). All the evidence ( $a b$ initio gene models, spliced transcript alignments, spliced protein alignments, ORFs, and BUSCO) was combined to consensus, high-confidence gene models, using EVidenceModeler (v1.1.1). This was followed by the addition of untranslated regions (UTR) to the gene models by PASA (Brian J. Haas et al., 2008).

Mitochondrial genome was also assembled and annotated as described previously (Salojärvi et al., 2017), resulting in 29 tRNAs, 32 coding sequences, and 3 rRNAs. Interproscan (v5.25-64.0) (Quevillon et al., 2005) was used to assign the protein function to gene models. Additionally, Ensemble Enzyme Prediction (E2P2, v3.1) (Schlapfer et al., 2017) and antiSMASH (v2.0) fungal version (Blin et al., 2013) were used to predict the metabolomic pathways. 
bioRxiv preprint doi: https://doi.org/10.1101/2021.11.28.470225; this version posted November 28,2021 . The copyright holder for this preprint (which was not certified by peer review) is the author/funder, who has granted bioRxiv a license to display the preprint in perpetuity. It is made available under aCC-BY-NC-ND 4.0 International license.

\section{Comparative genomic analyses}

The proteomes of twenty fungal species Laccaria bicolor, Coprinopsis cinerea, Schizophyllum commune, Fomitiporia mediterranea, Inonotus obliquus, Onnia scaura, Phellinidium ferrugineofuscum, Porodaedalea niemelaei, Trichaptum abietinum, Rickenella mellea, Schizopora paradoxa, Fomitopsis betulina, Postia placenta, Phanerochaete chrysosporium, Puccinia graminis, Heterobasidion annosum, Ustilago maydis, Saccharomyces cerevisiae, Schizosaccharomyces pombe, and Neurospora crassa from Ascomycete and Basidiomycete clades were downloaded from MycoCosm (https://mycocosm.jgi.doe.gov) and included for gene family analysis by Orthofinder (Emms \& Kelly, 2015) (v2.3.3), run with default parameters.

\section{Synteny analyses}

Synteny analysis of self-self alignment of $I$. obliquus, and four other fungal species, namely $F$. mediterranea, S. paradoxa, F. betulina, and P. niemelaei, were conducted using SynMap application in CoGe platform (https://genomevolution.org/coge/), using Quota Align algorithm with default parameters. The list of syntenic duplicates were obtained from DAGchainer (B. J. Haas, Delcher, Wortman, \& Salzberg, 2004); tandem duplicates were obtained as part of the preprocessing pipeline.

\section{Discovery of secreted proteins and carbohydrate active enzymes (CAZymes)}

Getorf function of EMBOSS (v6.6.0.0) (Rice et al., 2000) was used to discover the ORFs (-find:1, and-maxsize: 1000). All the ORFs were analyzed by signalp (v5) (Almagro Armenteros et al., 2019) for the presence of signal peptide. Signal peptides were removed from predicted ORF sequences, and the cysteine amino acids were counted for every sequence. ORF sequence with three cysteine residues was predicted as a possible secreted protein (SP).

CAZymes are annotated during gene model annotation steps. In order to further classify these enzymes, total proteome of $I$. obliquus was queried against dbCAN2 database using DIAMOND (v0.9.24, blastp, --more-sensitive) (Buchfink et al., 2014; H. Zhang et al., 2018), and the best hit was selected (score $\geq 200$, percentage identity $\geq 55$ ) as a homologous sequence.

\section{Gene tree of cytochrome P450 monooxygenases}

A phylogenetic tree was constructed for 15 CYP716 gene models from Streptophyta species which have been confirmed to produce betulinate compounds [Betula pendula (Salojärvi et al., 2017), Betula platyphylla, Phoenix dactylifera (Al-Mssallem et al., 2013), Medicago truncatula (Tang et al., 2014), and Vitis vinifera (The French-Italian Public Consortium for Grapevine Genome et al., 2007)], after which CYP450 genes with high sequence similarity $(<40 \%)$ in I. obliquus and $F$. betulina were added to the tree. A total of 77 sequences were subjected to multiple sequence alignment (MSA) 
using MUSCLE (v3.8.31, -maxiters: 1000) (Edgar, 2004). The amino acid sequences were reverse translated by PAL2NAL (Suyama, Torrents, \& Bork, 2006), and both amino acid and nucleotide sequences were used to construct the phylogenetic trees by RaxmIHPC-HYBRID-AVX2 (v8.2.12) (Stamatakis, 2014).

\section{Mass spectrometry, sample preparation and derivatization of $I$. obliquus and $F$.} mediterranea metabolites

Five strains of $I$. obliquus and one F. mediterranea (three biological replicates for each) were grown in liquid Hagem media for two weeks. Submerged myceliums were washed with sterile milli-

Q water three times, and grinded with liquid nitrogen. All samples (fresh wight $\sim 500 \mathrm{mg}$, dry weight 30 mg) were extracted twice with $1.0 \mathrm{ml}$ of ethyl acetate (Merck) by vortexing for 15 min and centrifuged for $10 \mathrm{~min}$ in $15000 \mathrm{rpm}$ according to Cao et al. (G. Zhao, Yan, \& Cao, 2007) at room temperature. Internal standards (ISTD) $(10 \mu \mathrm{l}, 10 \mu \mathrm{g} / \mathrm{ml})$, testosterone and 4-methylumbelliferone (Sigma), were added to each sample in the first extraction step. The supernatant was evaporated to dryness with MiVac Duo concentrator $+40^{\circ} \mathrm{C}$ (GeneVac LtD, Ipswich, UK) and the residue was resolubilized in $100 \mu \mathrm{ACN}$ (Honeywell). Quality control (QC) sample was prepared by combining extracts from each sample line.

The triterpenoid profiling was executed from the extracts with UPLC-PDA-QTOF/MS. The UPLCMS system consisted of a Waters Acquity UPLC attached to a Acquity PDA-detector and to a Waters Synapt G2 (HDMS) QTOF mass spectrometer (Waters, Milford, MA, USA). The separation of the analytes was executed in Acquity BEH C18 $(2.1 \mathrm{~mm} \times 50 \mathrm{~mm}, 1.7 \mu \mathrm{m})$ column (Waters, Milford, MA, USA) with the temperature of $+40^{\circ} \mathrm{C}$. The autosampler temperature was set to $+27^{\circ} \mathrm{C}$. The mobile phase consisted of water $(A)$ and acetonitrile (B) both with $0.1 \%$ formic acid and the flow rate was $0.6 \mathrm{ml} / \mathrm{min}$. The injection volume was $3 \mu \mathrm{l}$. The linear gradient started with $30 \% \mathrm{~B}$ and proceeded to $98 \%$ in $9 \mathrm{~min}$, followed by $1 \mathrm{~min}$ at $98 \% \mathrm{~B}$, giving a total run time of $10 \mathrm{~min}$. ESI/MS detection was performed in positive sensitivity ion mode with capillary voltage $3.0 \mathrm{kV}$, cone voltage $30 \mathrm{~V}$, desolvation gas $800 \mathrm{~L} / \mathrm{h}$, cone gas $20 \mathrm{~L} / \mathrm{h}$, desolvation temperature $320^{\circ} \mathrm{C}$, source temperature 120 ${ }^{\circ} \mathrm{C}$ and extractor lens 3.00 V. The MarkerLynx software (Waters, Milford, MA, USA) was used for data processing. UV spectra, negative MS-runs and fragmentation patterns from $\mathrm{MS}^{\mathrm{e}}$ runs of QC sample were used as additional tools for annotation of triterpenes, sterols and phenolic compounds in Inonotus obliquus samples.

The standard solutions of betulin and betulinic acid $(1.0 \mathrm{mg} / \mathrm{ml})$ were prepared in ethyl acetate and testesterone $(100 \mu \mathrm{g} / \mathrm{ml})$ standard was prepared in methanol. Working solutions $(10 \mu \mathrm{g} / \mathrm{ml}, 100$ $\mathrm{ng} / \mathrm{ml}$ ) were prepared by diluting the standard solution with acetonitrile. The optimization of quantification method was executed with a mix of betulin, betulinic acid and testosterone standards 
(100 ng/ml). Standard mix $(100 \mu l)$ was derivatized with PTSI, and MS parameters were optimized by repeated injection of the sample.

Due to very low concentration of betulin and betulinic acid, extracts were derivatized with $p$ toluenesulfonyl isocyanate (PTSI) (Hu et al., 2013; Zuo, Gao, Liu, Cai, \& Duan, 2005) to improve sensitivity. After metabolite profiling with UPLC-QTOF/MS, samples (90 $\mu$ l) were derivatized for 3 min with $10 \mu \mathrm{l}$ of $60 \%$ p-toluenesulfonyl isocyanate (PTSI) (Sigma) in acetonitrile (Hu et al., 2013; Zuo et al., 2005). The derivatization reaction was terminated with $50 \mu$ l of methanol (Merck) with $30 \mathrm{~s}$ vortex mixing, giving the total volume of $150 \mu \mathrm{l}$.

The UPLC-MS/MS system consisted of a UPLC (ABSciex, Shimadzu) attached to ABSciex 6500+ QTRAP mass spectrometer with ESI source. The Acquity BEH C18 $(2.1 \mathrm{~mm} \times 50 \mathrm{~mm}, 1.7 \mu \mathrm{m})$ (Waters, Milford, MA, USA) column was used for the separation of compounds, and column oven temperature was $+40^{\circ} \mathrm{C}$. The autosampler temperature was set to $+25^{\circ} \mathrm{C}$. Injection volume was $2 \mu$. rate was $0.6 \mathrm{ml} / \mathrm{min}$. The linear gradient started from $30 \% \mathrm{~B}$ and proceeded to $98 \%$ in $6.5 \mathrm{~min}$, followed by $1.5 \mathrm{~min}$ at $98 \% \mathrm{~B}$, giving a total run time of $8 \mathrm{~min}$. The data was normalized to dry weight (DW) and to the peak area of internal standard. The Analyst software (ABSciex) was used for data processing and quantification.

PTSI derivatization reagent generated betulin p-toluenesulfonyl carbamic diester, betulinic acid p-toluenesulfonyl carbamic ester, and testosterone toluenesulfonyl carbamic ester. Two MRM transitions were selected for each analyte, one for quantification and other for qualification. The ratio between quantification (quan) and qualification (qual) transitions should stay stable among runs. The transitions were as follows: betulin MRM $835.3 \rightarrow 620.3$ quan [M-PTSI- $\mathrm{H}_{2} \mathrm{O}-\mathrm{H}$ ]', $835.3 \rightarrow$ 638.3 qual [M-PTSI-H]', betulinic acid MRM 652.3 $\rightarrow 455.2$ quan [M-PTSI-H] $652.3 \rightarrow 437.2$ qual [MPTSI- $\mathrm{H}_{2} \mathrm{O}-\mathrm{H}^{-}$, and testosterone $484.2 \rightarrow 287.2$ quan [PTSI-H]', $484.2 \rightarrow 269.2$ qual [PTSI- $\left.\mathrm{H}_{2} \mathrm{O}-\mathrm{H}\right]^{-}$. ESI source temperature was set to $450{ }^{\circ} \mathrm{C}$. ESI/MS/MS detection was performed in negative ion mode with ion spray (IS) voltage of -4000 , curtain gas (CUR) 30, collision gas (CAD) at medium, entrance potential (EP) -10, declustering potential (DP) -60 (betulinic acid, testosterone) or -100 (betulin), collision energy (CE) -50, collision cell exit potential (CXP) -10.

Cloning and mass spectrometry of lupeol synthase and CYP450 monooxygenase enzymes

Two major cloning constructs were designed for betulin biosynthesis using pRS424 vector (Burgers, 1999). This version of pRS424 vector contained two multiple cloning sites (MCS), one under 
monooxygenases from I. obliquus (pRS424::CYP450). The second construct was a double insertion (pRS424::LUS-CYP716) of lupeol synthase (Bpev01.c0219.g0020.m0001 under GAL10 promoter) and CYP450 monooxygenase (Bpev01.c0219.g0021.m0001, under GAL1 promoter) enzymes isolated from $B$. pendula in pRS424 vector. All the vector constructs were transformed to yeast strain (Saccharomyces cerevisiae [w303 background]). The transgenic yeasts were grown and induced according to Zhou et al. (Zhou, Li, Li, \& Zhang, 2016), with minor changes. SD-TRP was us as the drop out medium. For single inserted vectors, we used 50um lupeol (dissolved in DMSO:EtOh [1:1]) in induced growth media. After 60 hours of induction, the yeast growth media were centrifuged, and both media and the cell pallets were collected and sent for mass spectrometry.

Total of eight samples (yeast cells (4 tubes), and cell culture media (4 tubes)) were analyzed with UPLC-QTRAP/MS (MRM). Three triterpenoids (betulin [BE]) were extracted first from the media twice with $1.0 \mathrm{ml}$ ethyl acetate (Merck) for $60 \mathrm{~min}$ in RT and centrifuged for $5 \mathrm{~min}$ in $15000 \mathrm{rpm}$ according to Cao et al. (G. Zhao et al., 2007). Testosterone was used as an internal standard (ISTD, $1.0 \mu \mathrm{l}, 1.0 \mu \mathrm{g} / \mathrm{ml}$ ). The cells were extracted in a similar manner as media, but yeast cells with $500 \mu \mathrm{l}$ $\mathrm{H}_{2} \mathrm{O}$ and $1000 \mu \mathrm{l}$ chloroform twice and were disrupted with freeze/thaw cycle (3 cycles) with ultrasonication (15min) prior to extraction procedure.

The upper ethyl acetate was evaporated to dryness with MiVac Duo concentrator $+40^{\circ} \mathrm{C}$ (GeneVac Ltd., Ipswich, UK). The residue was re-solubilized in $100 \mu \mathrm{l}$ ACN. Due to very low concentration of lupeol, betulin and betulinic acid, extracts had to be derivatized with $p$ toluenesulfonyl isocyanate (PTSI) (Hu et al., 2013; Zuo et al., 2005) to improve sensitivity. Samples were derivatized in RT for $3 \mathrm{~min}$ with $10 \mu \mathrm{l}$ of $60 \%$ p-toluenesulfonyl isocyanate (PTSI) (Sigma Aldrich) in ACN (Hu et al., 2013; Zuo et al., 2005). The derivatization reaction was terminated with $90 \mu \mathrm{l}$ of $\mathrm{MeOH}$ with $30 \mathrm{~s}$ vortex mixing, giving the total volume of $200 \mu \mathrm{l}$. Immediately after PTSIderivatization, the MRM analysis of lupeol, betulin and betulinic acid was executed with UPLCQTRAP/MS (ABSciex). spectrometer. The separation of the analytes column was Acquity BEH C18 $(2.1 \mathrm{~mm} \times 50 \mathrm{~mm}, 1.7 \mu \mathrm{m})$ (Waters, Milford, MA, USA), with the temperature of $+40^{\circ} \mathrm{C}$. The autosampler temperature was set to $+25^{\circ} \mathrm{C}$. The injection volume was $10 \mu \mathrm{l}$. The chromatographic conditions were executed as described previously at Hua et al. (Hu et al., 2013). The mobile phase consisted of water with $0.1 \%$ of formic acid in $\mathrm{H}_{2} \mathrm{O}(\mathrm{A})$ and acetonitrile (B) with a flow rate of $0.6 \mathrm{ml} / \mathrm{min}$. The linear gradient started 30\% B and proceeded to $98 \%$ in $6.5 \mathrm{~min}$, left in $98 \%$ B for $2 \mathrm{~min}$, and switched back to initial conditions and left to stabilize, giving a total analysis time of $10 \mathrm{~min}$. 
$\mathrm{ESI}$ source temperature was set to $450^{\circ} \mathrm{C}$. $\mathrm{ESI} / \mathrm{MS} / \mathrm{MS}$ detection was performed in negative ion mode with ion spray (IS) voltage of -4000 , curtain gas (CUR) 30, collision gas (CAD) at medium, entrance potential (EP) -10, de-clustering potential (DP) -60 (betulinic acid, testosterone) or -100 (betulin), collision energy (CE) -50 , collision cell exit potential (CXP) -10 . The Analyst software (ABSciex) was used for data processing. PTSI derivatization reagent generated betulin $p$ toluenesulfonyl carbamic diester (BTCD). The transitions for betulin and ISTD (testosterone) was as

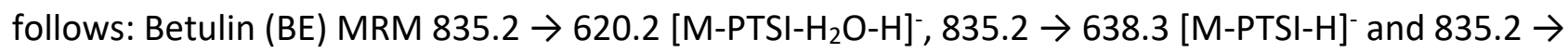
196.0 [PTSI-H]', and for testosterone MRM 484.2 $\rightarrow 287.2$ [M-PTSI-H] $^{-}$and MRM 484.2 $\rightarrow 269.2$ [M- $^{-}$ PTSI- $\left.\mathrm{H}_{2} \mathrm{O}-\mathrm{H}\right]^{-}$. The most intense transitions of MRM $835.2 \rightarrow 620.2$ (betulin), and MRM $484.2 \rightarrow$ 287.2 (ISTD, testosterone) were used.

\section{Results and discussion}

\section{Nuclear and mitochondrial genome assemblies and annotations}

374 Pacbio sequencing of $I$. obliquus strain from Merikarvia yielded $4.82 \mathrm{~Gb}$ of data (96x coverage) 375 with $\mathrm{N} 50$ read length of 9,200 bp. Falcon assembly resulted in a 41.1 million bp genome, consisting 376 of 301 primary contigs with an N50 value of 516 kilobases. Overall, the genome size of I. obliquus was comparable with other species from Hymenochaetales (Supp. table 1).

Genome annotation of primary assembly yielded 13,778 gene models with $91.7 \%$ of universally conserved single-copy genes being present (BUSCO v3.0, fungi database) (Waterhouse et al., 2017). To support gene model prediction, RNA-seq was carried out from total RNA extracted from $I$. obliquus reference strain sample grown on wood dust. Genome-guided de novo assembly of RNAseq data showed 91.3\% BUSCO completeness with 31\% duplicated genes (Supp. table 1). Altogether $70.8 \%$ of $I$. obliquus genome consisted of coding sequence, with $53.1 \%$ in exons. Mean intron, exon, CDS and gene lengths were 89, 284, 1447, and 2113 base pairs, respectively (Supp. table 1). Mitochondrial genome was also assembled and annotated as described previously (Salojärvi et al., 2017), resulting in 29 tRNAs, 32 coding sequences, and 3 rRNAs.

Transposable elements (TEs) have been suggested to play a major role in genome plasticity and evolution. Thus, the classification and characterization of genes in close proximity of TEs are of general interest, especially in the case of pathogenic organisms (Faino et al., 2016). In I. obliquus, the total genome repeat content was found to be $26 \%$, with $14.24 \%$ of repeats being unclassified. The percentage of retrotransposon elements was $8.37 \%$, and DNA transposon elements $1.2 \%$. In contrast to retrotransposon elements, I. obliquus genome contained higher amounts of DNA transposon elements compared to the related F. betulina and F. mediterranea (Supp. table 1). Unlike F. mediterranea (42.27\% repeat sequences), TE content of I. obliquus did not fully explain its large 
genome size (26\% of repeat sequences) (Hage et al., 2021). Gene models flanking the upstream and

downstream of TEs contained mainly transposition elements, and gene clusters between two transposable elements from the same DNA transposon class suggested the enrichment of gene models involved in transmembrane transporter (GO:0003677), protein dimerization (G0:0046915), transposition (GO:0046983 and GO:0006310), and DNA biding and recombination (GO:0006313 and GO:0032196) (Supp. table 1) (Ali et al., 2014; Kang, Lebrun, Farrall, \& Valent, 2001). The enrichment

of the last two categories suggests that some of the predicted gene models may be unidentified transposable elements, and they are organized as clusters in the genome.

\section{Secreted proteins in I. obliquus}

The exact mechanisms of $I$. obliquus pathogenicity and its modes of interaction with the host are not known, but characterization of secreted proteins is the first step to shed more light on the

mechanisms involving the initial penetration of plant defences by effector proteins. Secreted proteins (SPs) are known for their essential role in pathogen-host interactions. Altogether 1052 open reading frames (ORFs), $7.6 \%$ of all gene models, were predicted as possible secreted proteins, with minimal known homologs (Supp. table 2). The SPs were scattered across 128 contigs. Most of

411 other species were successful for only 110 of the ORFs (Supp. table 2). Twenty-one ORFs overlapped 412 at least with one class of TEs, having eighteen unclassified categories. Total of 988 ORFs were co413 localized between two TEs of the same TE class, suggesting a role for TEs in SP evolution and 414 diversification.

\section{Carbohydrate-active enzymes}

416 The palette of carbohydrate-active enzymes (CAZymes) present in the genome dictate to a large 417 extent the modes of substrate utilization by the fungus (Eastwood et al., 2011; Navarro et al., 2021). 418 We identified 466 candidate genes classified as carbohydrate-active enzymes (CAZymes), known for 419 their biological roles in anabolism and catabolism of different carbohydrates such as glycogen, 420 trehalose, and glycoconjugates (Supp. table 5). Altogether 211 enzymes were classified as glycoside 421 hydrolases (GHs) and 43 were categorized as carbohydrate binding modules (CBMs) whereas the 422 overall number of glycosyltransferases (GTs) and carbohydrate esterases (CEs) were found to be 110 423 and 23, respectively. Finally, 10 enzymes were assigned to polysaccharide lyases (PLs) (Supp. table 424 5). Overall the CAZyme palette was similar to other lignin-degrading fungi (Liu et al., 2019). RNAseq 425 analysis of $I$. obliquus grown on B. pendula wood dust and publicly available RNAseq data (Fradj et 
of the experimental conditions. Majority of DE CAZymes belonged to glycoside hydrolases and glycosyltransferases categories.

\section{Phylogenomics and expanded gene families in I. obliquus genome}

In order to estimate a taxonomic placement for I. obliquus, we collected the proteomes of fifteen representative fungal species from different orders among Basidiomycota: all sequenced species within Hymenochaetales (Fomitiporia mediterranea, Inonotus obliquus, Onnia scaura, Phellinidium ferrugineofuscum, Porodaedalea niemelaei, Trichaptum abietinum, Rickenella mellea, Schizopora paradoxa), and representatives of Russulales (Heterobasidion annosum), Polyporales (Fomitopsis

435 betulina, Postia placenta, Phanerochaete chrysosporium) and Agaricales (Laccaria bicolor, 436 Coprinopsis cinerea, Schizophyllum commune). To root the taxonomy we added five outgroup 437 species, including two representatives of the other major classes in Basidiomycota: Pucciniales 438 (Puccinia graminis) and Ustilaginales (Ustilago maydis), as well as three model Ascomycota species 439 from Saccharomycetales (Saccharomyces cerevisiae), Schizosaccharomycetales 440 (Schizosaccharomyces pombe), and Sordariales (Neurospora crassa). We next clustered the full 441 proteomes into gene families (orthogroups) using Orthofinder and identified single copy 442 orthogroups. Phylogeny estimation was carried out using 4040 single copy gene groups and rooted 443 to Ascomycota species. The resulting tree illustrates the known taxonomy among the 20 fungal 444 species (Figure 1; Supp. table 3) and the split of Hymenochaetaceae family occurs at the expected 445 phylogenetic position (Hibbett \& Thorn, 2001; Matheny et al., 2007; R.-L. Zhao et al., 2017) (Figure 446 1). Interestingly $R$. mellea was placed together with the Russulales representative, further analysis 447 is however beyond the scope of the present work.

448 To look for gene family evolution we then identified gene families that were expanded in $I$. 449 obliquus. Altogether 167 orthologous gene clusters were significantly expanded in comparison to 450 the other nineteen fungal species (chi-squared test; Supp. table 4Error! Reference source not 451 found.). The expanded gene families were enriched for $23 \mathrm{GO}$ terms such as terpene synthase 452 (GO:0010333), oxidoreductase (GO:0016684), and hydrolase (GO:0016788) activities. In addition, 453 GOs related to oxidative stress responses (GO:0006979), transposition (GO:0015074, GO:0006313), 454 and protein dimerization activity (GO:0046983) were also expanded (Supp. table 4); most of these categories involve members of cytochrome P450 gene family.

\section{Genome evolution in I. obliquus}

457 The high-quality whole genome assembly allowed us to gain further insight into the gene family 
genome duplication (WGD) event in I. obliquus. Based on the synonymous mutation (Ks) spectrum the event occurred after the split from F. mediterranea (approximately 112 million years ago during the Triassic period; (Kumar, Stecher, Suleski, \& Hedges, 2017)). Similarly, an independent lineagespecific WGD was observed also in P. niemelaei (Figure 2).

Synteny analysis identified a total of 1,112 genes originating from the whole genome duplication event, whereas a considerably higher amount, 6,200 genes, were identified in tandem duplications (Supp. table 3). The tandemly duplicated genes reflect the shorter-term adaptation in the species, and in general have been found to be associated with environmental responses (Panchy, Lehti-Shiu, \& Shiu, 2016). In I. obliquus, the tandemly duplicated genes were enriched for carbohydrate biosynthesis, heme binding, oxidoreductase activity, tetrapyrrole binding, and DNA transposition. In contrast, syntenic regions harbored genes related to biological pathways such as terpene synthesis and cell cycle (Figure 3, Supp. table 6). The overlaps between tandemly duplicated, syntenic genes and expanded gene families were significant ( $p$-value=6.1094e-11, Fisher exact test) (Figure 4). Altogether, the genome evolution analyses highlight the significance of tandem duplication events in adaptation of the $I$. obliquus to different ecological niches and the central role of secondary metabolism and particularly the expansion of CYP450 gene family by small scale duplication events (Figure 5).

The members of CYP450 family have critical roles in fungal metabolism and adaptation to specific ecological niches. Altogether 172 CYP450 monooxygenases were predicted in chaga, suggesting a complex biochemical diversity in chaga metabolism. Division into clans and families revealed that most of the enzymes belonged to clan CYP620 (69 members), followed by CYP4 and CYP512 clans (Figure 5). CYP620 is shown to be involved in terpenoid synthases (Yap et al., 2014; Yu, Song, Liang, Wang, \& Lu, 2020). CYP4 is studied predominantly in phylum of Arthropoda, and shown to be involved in biosynthesis of endogenous compounds (Zhu, Moural, Shah, \& Palli, 2013). Finally, CYP512 clan has been hypothesised to have catalytic activities towards steroidal-like compounds, primarily testosterone (Ide, Ichinose, \& Wariishi, 2012).

A high proportion CYP450 gene models, 79 out of the total of 172, were tandemly duplicated, and many were members of CYP620 clan. GO enrichment analysis of two genes upstream and two genes in downstream of all CYP450 monooxygenase enzymes suggested the enrichment of biological functions related to oxidoreductase activity, heme binding, transmembrane transporter activities, and tetrapyrrole binding (Supp. table 6). These results suggest tandem duplications of CYP450s, and additionally the colocalization with cytochrome P450 reductase (CPR) partners, facilitates their functional divergence (Ebrecht et al., 2019). 
The observed colocalization of genes related to CYP450s suggested the presence of biosynthetic clusters in the I. obliquus genome. We therefore sought biosynthetic gene clusters by antiSMASH

494 (Blin et al., 2013), identifying altogether 24 clusters in 17 contigs: 15 terpene synthase, 3 polyketide synthase, and 4 non-ribosomal peptide synthetase clusters. The clusters were significantly enriched for tandemly duplicated genes (Fisher exact test, p.value=6.34E-76), suggesting that tandem duplications are a dominant process in their diversification. Furthermore, the clusters were enriched for CYP450 gene family ( $p$-value: 0.03161975), highlighting their central enzymatic role in secondary metabolism (Supp. table 6).

Metabolomics fingerprinting of terpenoid compounds in five $I$. obliquus strains and $F$. mediterranea

Since chaga showed a significant expansion of CYP450 genes and a considerable number of biosynthetic clusters, we next carried out metabolic fingerprinting of $I$. obliquus to study whether the secondary metabolism was indeed diversified in chaga compared to F. mediterranea. Terpenoid fingerprints in five strains of $I$. obliquus were distinctly different from F. mediterranea (Supp. table 1). Altogether the chaga strains showed 546 mass spectrum peaks, and only 135 of them were shared with F. mediterranea (Figure 6 A). In addition, pairwise comparisons of each chaga strain and F. mediterranea found 178 metabolomic features among I. obliquus strains with significantly higher abundance (Supp. Fig 2, Supp. table 7). Many of the peaks were predicted to have molecular formulae with 30,31 , and 28 carbon backbones, similar to lupeol, betulin and betulinic acid. Among I. obliquus strains, Merikarvia had distinct metabolomic fingerprints and clustered more distant from 513 plays a role in metabolic diversity (Figure $6 \mathrm{C}$ ). With regards to betulinate compounds, Merikarvia 514 strain had higher abundance of betulin and betulinic acid production in comparison to other strains 515 of I. obliquus. There were no peaks which resembled the standard for betulin or betulinic acid (98\%, 516 Sigma-aldrich) in F. mediterranea, but a significant quantity of lupeol-like substance was discovered 517 (Supp. Fig 2).

\section{Functional analysis of lupeol synthase and CYP450 monooxygenase}

Even though metabolic fingerprinting does not identify the underlying metabolites, the analysis suggested the presence of terpene and lupeol as well as betulin derivatives based on the predicted carbon backbones. Intraspecies quantification of betulin and betulinic acid (Using HPL) among six species of betula and three strains of chaga showed a higher concentration of betulinic acid compared to betulin in chaga strains. In contrast, the opposite result was observed in six species of 
524 Betula, where the concentration of betulin was consistently higher in comparison to betulinic acid 525 (Figure 7).

The high quality gene model predictions allowed us to look for the candidate enzymes responsible for the betulin biosynthesis in chaga. Since no members of CYP716 family were not predicted in chaga we identified four best candidates based on homology analysis of CYP450s to known CYP716 family members from plant species, such as $B$. pendula. Yeast expression system has

530 been used successfully for cloning CYP450 monooxygenase enzyme from B. platyphylla (Zhou et al., 531 2016). To functionally validate our candidate genes, we first constructed single insert expression 532 vector for birch CYP716 enzyme (pRS424::CYP716). In addition, we also constructed a double insert 533 vector where lupeol synthase and lupeol monooxygenase from B. pendula (Safronov et al., 2019) 534 were inserted into two multiple cloning sites of a vector (pRS424::LUS-CYP716), which was then 535 transferred into yeast expression system. Similar to single insert vector for CYP450 monooxygenase 536 enzyme from B. pendula, we isolated four CYP450s from I. obliquus and cloned them to engineer 537 single inserted constructs. The single constructs were grown in media which was spiked with 538 standard lupeol compound (98\%, Cayman) as the precursor. In contrast to single insert vectors, 539 double insert vector from B. pendula (pRS424::LUS-CYP716) expressed lower concentration of 540 betulin compared to single insert vector (pRS424::CYP716) from B. pendula. These differences might 541 be explained by the lower initial amount of available precursor compound, lupeol, for CYP716 542 monooxygenase (Figure 8). In addition to B. pendula constructs, all four candidate CYP450 543 monooxygenases from I. obliquus (pRS424::CYP450) showed some degree of betulin production 544 when compared to standard betulin (98\%, Sigma-Aldrich) spectrum. Among the four candidates, the 545 enzyme with gene ID c000016F_g277 (clan CYP505) had the highest amount of betulin production. 546 Interestingly, the cDNA length of this enzyme was $3297 \mathrm{bp}$, almost twice the length of the other 547 three candidate CYP450 monooxygenase homologs from I. obliquus. Upon close examination the 548 amino acid and nucleotide sequences of c000016F_g277 resemble a chimeric isoform of two CYP450 549 monooxygenase enzymes (Figure 8). In general, our study showed that yeast cell fractions contained 550 higher concentration of betulin compared to the culture media fractions (Figure 8), thus confirming 551 the function of the inserted enzymes.

552 To study the evolution of potential homologs and orthologs for the four candidate genes from $I$. 553 obliquus, we carried out microsynteny analysis for the cloned CYP450 monooxygenase enzymes 554 against four Hymenochaetales and F. betulina species. Orthologous one-to-one relationship with 555 other fungal species was confirmed for c000112F.g25 (clan CYP51) and c000041F.g53 (clan CYP51) 556 enzymes (Supp. Fig 3-C,D), whereas microsynteny analysis of c000000F.g253 (clan CYP61) enzyme 557 found a cluster of homologous genes in 5' and $3^{\prime}$ of the c000000F.g253 in I. obliquus genome (Supp. 
Fig 3-B). The microsynteny of the chimeric c000016F.g277 linked to a putative ortholog in F. mediterranea with similar organization (gene_7933, clan CYP505) (Supp. Fig 3Error! Reference source not found.-A), whereas in other Hymenochaetales the syntenic analysis identified two separate CYP450s. This suggests that the fusion gene has arisen from a non-homologous recombination event in the common ancestor of F. mediterranea and I. obliquus, and after divergence of $P$. niemelaei where the CYP450s were still found separate. Both c000016F.g277 and gene_7933 contain two heme-binding domains, but gene_7933 from F. mediterranea has three oxygen-binding domains (with AGADTT/GGDDTG motifs) instead of two in I. obliquus (AGADTT). Therefore the fusion may have occurred also independently in chaga and F. mediterranea (Supp. table 8), or then involved a loss of the third oxygen-binding domain in chaga.

\section{Evolution of conserved domains and phylogeny reconstruction of cytochrome P450 monooxygenase}

Since betulin and betulinic acid are antifungal substances and they are produced in the main natural host of $I$. obliquus, it is possible that the enzymes have been introduced into chaga or its ancestor via horizontal gene transfer, either directly from the host species or then via another species cohabiting with chaga. However, a phylogenetic tree of a set of monooxygenase enzymes (77 enzymes) from I. obliquus and F. betulina with sequence similarity to plant CYP716, as well as CYP716 enzymes of eight plant species known to produce betulinate compounds, shows a distinct divergence of fungal clades from the plant species. This result is consistent both in protein and DNA based phylogenetic trees, providing no evidence of gene transfer events (Figure 9).

\section{Gene expression analysis}

Total of 119 (out of 172) monooxygenase enzymes were significantly expressed with positive $\log _{2}$ fold change $\left(\log _{2} \mathrm{FC}\right)$ values in at least one of the DEg comparisons (Figure 5 ) and three key enzymes involved in mevalonate pathways were among this set (Error! Reference source not found.). We also observed a pair of tandemly duplicated lupeol synthase enzymes to have the highest expression levels. The expression profiles of the chaga samples grown on B. pendula wood dust were stronger than the samples grown in culture media from (Fradj et al., 2019). When inspecting the expression profiles for genes with positive $\log _{2} \mathrm{FC}$, enrichments were found for WD40-repeat binding (50 genes out of 294), melanin biosynthesis (65 genes out of 157), aquaporin (20 genes), lipases and peptidases (Supp. table 9). 
bioRxiv preprint doi: https://doi.org/10.1101/2021.11.28.470225; this version posted November 28, 2021. The copyright holder for this preprint (which was not certified by peer review) is the author/funder, who has granted bioRxiv a license to display the preprint in perpetuity. It is made available under aCC-BY-NC-ND 4.0 International license.

\section{Conclusion}

We observed genome evolution leading towards complex terpene biosynthesis in I. obliquus, both in genes originating from whole genome duplication events as well as tandem duplications within the CYP450 gene family. It is possible that the whole genome duplication event is associated with the initial expansion of terpenoid biosynthesis capacity in I. obliquus, since no such expansion was observed in the related species F. mediterranea. In contrast to eg plants, the number of whole genome duplication events in fungal kingdom has been low (Albertin \& Marullo, 2012), but this may be due to faster genome evolution in fungi, making the WGDs difficult to identify (Campbell, Ganley, Gabaldón, \& Cox, 2016). The CYP450 superfamily is associated with many reactions in secondary metabolism, and through metabolomics fingerprinting we confirmed that the fungus indeed produces a rich palette of terpenoid derivatives. However, we found no evidence of a horizontal gene transfer event between $B$. pendula and $I$. obliquus, and the identified candidate lupeol monooxygenases in I. obliquus were members of a different CYP505 clan with low sequence similarity to their birch counterpart enzymes. Therefore CYP450 monooxygenases enzymes responsible for betulinate biosynthesis in the two species most likely result from convergent evolution.

\section{Author's contributions}

O.S and J.S conceived and designed the project. Funding acquisition is carried by J.S and J.K. O.S collected the DNA and RNA samples. O.S and J.S managed and coordinated all bioinformatics activities. O-P.S, L.G.P, and P.A did RNA and DNA library construction and sequencing and participated in genome assembly. O.S did the genome and functional annotation. S.R and P.S participated in genome annotation. O.S analyzed the RNA sequencing data including de novo assembly of RNAseq. O.S did comparative genomics analyses. T.S and N.S were involved in field research for sample isolations. O.S and M.W grown and collected the samples for mass spectrometry. G.L.B, B.B, M.W, and O.S were sequenced were involved in cloning and expression of CYP450 and Lupeol synthase enzymes. N.S and J.L did mass spectrometry, including sample pretreatment, method development, UPLC-HDMS analysis, metabolite identification and data interpretation, and O.S and J.S contributed to data interpretation. O.S and J.S wrote the original manuscript with input from O-P.S, U.R, K.O.

\section{Acknowledgement}

We thank Cory D. Dunn who provided us with yeast strain, and Peter M.J. Burgers, Ville O. Paavilainen, and Juho Kellosalo for giving us the expression vector. We also acknowledge the computational infrastructure of CSC IT Center for Science, Finland. J.S would like to acknowledge 
bioRxiv preprint doi: https://doi.org/10.1101/2021.11.28.470225; this version posted November 28, 2021. The copyright holder for this preprint (which was not certified by peer review) is the author/funder, who has granted bioRxiv a license to display the preprint in perpetuity. It is made available under aCC-BY-NC-ND 4.0 International license.

the funding from University of Helsinki three-year grant, Academy of Finland (decisions 318288 and 319947), as well as Nanyang Technological University start-up grant.

\section{References}

Al-Mssallem, I. S., Hu, S., Zhang, X., Lin, Q., Liu, W., Tan, J., . . Yu, J. (2013). Genome sequence of the date palm Phoenix dactylifera $\mathrm{L}$. from Nat Commun

Albertin, W., \& Marullo, P. (2012). Polyploidy in fungi: evolution after whole-genome duplication. Proceedings of the Royal Society B: Biological Sciences, 279(1738), 2497-2509. doi:10.1098/rspb.2012.0434

Ali, S., Laurie, J. D., Linning, R., Cervantes-Chavez, J. A., Gaudet, D., \& Bakkeren, G. (2014). An immunity-triggering effector from the Barley smut fungus Ustilago hordei resides in an Ustilaginaceae-specific cluster bearing signs of transposable element-assisted evolution. PLoS Pathog, 10(7), e1004223. doi:10.1371/journal.ppat.1004223

Almagro Armenteros, J. J., Tsirigos, K. D., Sønderby, C. K., Petersen, T. N., Winther, O., Brunak, S., . .. Nielsen, H. (2019). SignalP 5.0 improves signal peptide predictions using deep neural networks. Nature Biotechnology, 37(4), 420-423. doi:10.1038/s41587-019-0036-z

Alresly, Z., Lindequist, U., Lalk, M., Porzel, A., Arnold, N., \& Wessjohann, L. A. (2015). Bioactive Triterpenes from the Fungus Piptoporus betulinus. Rec Nat Prod, 10, 103-108.

Andre, C. M., Larsen, L., Burgess, E. J., Jensen, D. J., Cooney, J. M., Evers, D., . . Laing, W. A. (2013). Unusual immuno-modulatory triterpene-caffeates in the skins of russeted varieties of apples and pears. J Agric Food Chem, 61(11), 2773-2779. doi:10.1021/jf305190e

Andrews, S. FastQC A Quality Control tool for High Throughput Sequence Data. http://www.bioinformatics.babraham.ac.uk/projects/fastqc/. Retrieved from http://www.bioinformatics.babraham.ac.uk/projects/fastqc/

Besemer, J., \& Borodovsky, M. (2005). GeneMark: web software for gene finding in prokaryotes, eukaryotes and viruses. Nuc acids res, 33. doi:10.1093/nar/gki487

Blanchette, R. A. (1982). Progressive stages of discolortion and decay associated with the cankerrot fungus, Inonotus obliquus, in birch. Phytopathology., 72(10), 1272-1277. doi:10.1094/phyto-72-1272

Blin, K., Medema, M. H., Kazempour, D., Fischbach, M. A., Breitling, R., Takano, E., \& Weber, T. (2013). antiSMASH 2.0--a versatile platform for genome mining of secondary metabolite producers. Nucleic Acids Res, 41(Web Server issue), W204-212. doi:10.1093/nar/gkt449

Bolger, A. M., Lohse, M., \& Usadel, B. (2014). Trimmomatic: a flexible trimmer for Illumina sequence data. Bioinformatics, 30(15), 2114-2120. doi:10.1093/bioinformatics/btu170

Bonfield, J. K., Smith, K., \& Staden, R. (1995). A new DNA sequence assembly program. Nucleic Acids Res, 23(24), 4992-4999.

Bray, N. L., Pimentel, H., Melsted, P., \& Pachter, L. (2016). Near-optimal probabilistic RNA-seq quantification. Nat Biotechnol, 34(5), 525-527. doi:10.1038/nbt.3519

Buchfink, B., Xie, C., \& Huson, D. H. (2014). Fast and sensitive protein alignment using DIAMOND. Nat Meth, 12, 59. doi:10.1038/nmeth.3176

https://www.nature.com/articles/nmeth.3176\#supplementary-information

Burgers, P. M. (1999). Overexpression of multisubunit replication factors in yeast. Methods, 18(3), 349-355. doi:10.1006/meth.1999.0796

Campbell, M. A., Ganley, A. R. D., Gabaldón, T., \& Cox, M. P. (2016). The Case of the Missing Ancient Fungal Polyploids. The American Naturalist, 188(6), 602-614. doi:10.1086/688763

Chang, S., Puryear, J., \& Cairney, J. (1993). A Simple and Efficient Method for Isolating RNA from Pine Trees. Plant Mol Biol Rep, 11(2), 113-116. doi:10.1007/BF02670468 
Chin, C. S., Peluso, P., Sedlazeck, F. J., Nattestad, M., Concepcion, G. T., Clum, A., . . Schatz, M. C. (2016). Phased diploid genome assembly with single-molecule real-time sequencing. Nat Methods, 13(12), 1050-1054. doi:10.1038/nmeth.4035

Eastwood, D. C., Floudas, D., Binder, M., Majcherczyk, A., Schneider, P., Aerts, A., . . Watkinson Sarah, C. (2011). The Plant Cell Wall-Decomposing Machinery Underlies the Functional Diversity of Forest Fungi. Science, 333(6043), 762-765. doi:10.1126/science.1205411

Ebrecht, A. C., van der Bergh, N., Harrison, S. T. L., Smit, M. S., Sewell, B. T., \& Opperman, D. J. (2019). Biochemical and structural insights into the cytochrome $P 450$ reductase from Candida tropicalis. Scientific Reports, 9(1), 20088. doi:10.1038/s41598-019-56516-6

Edgar, R. C. (2004). MUSCLE: multiple sequence alignment with high accuracy and high throughput. Nucleic Acids Res, 32(5), 1792-1797. doi:10.1093/nar/gkh340

Emms, D. M., \& Kelly, S. (2015). OrthoFinder: solving fundamental biases in whole genome comparisons dramatically improves orthogroup inference accuracy. Genome Biology, 16(1), 157. doi:10.1186/s13059-015-0721-2

Faino, L., Seidl, M. F., Shi-Kunne, X., Pauper, M., van den Berg, G. C., Wittenberg, A. H., \& Thomma, B. P. (2016). Transposons passively and actively contribute to evolution of the two-speed genome of a fungal pathogen. Genome Res, 26(8), 1091-1100. doi:10.1101/gr.204974.116

Fradj, N., Goncalves Dos Santos, K. C., de Montigny, N., Awwad, F., Boumghar, Y., Germain, H., \& Desgagne-Penix, I. (2019). RNA-Seq de Novo Assembly and Differential Transcriptome Analysis of Chaga (Inonotus obliquus) Cultured with Different Betulin Sources and the Regulation of Genes Involved in Terpenoid Biosynthesis. Int J Mol Sci, 20(18). doi:10.3390/ijms20184334

Fukushima, E. O., Seki, H., Ohyama, K., Ono, E., Umemoto, N., Mizutani, M., . . Muranaka, T. (2011). CYP716A subfamily members are multifunctional oxidases in triterpenoid biosynthesis. Plant Cell Physiol, 52(12), 2050-2061. doi:10.1093/pcp/pcr146

Godzik, A., \& Li, W. (2006). Cd-hit: a fast program for clustering and comparing large sets of protein or nucleotide sequences. Bioinformatics, 22(13), 1658-1659. doi:10.1093/bioinformatics/btl158

Gong, Y., Raj, K. M., Luscombe, C. A., Gadawski, I., Tam, T., Chu, J., . . Sacks, S. L. (2004). The synergistic effects of betulin with acyclovir against herpes simplex viruses. Antiviral Res, 64(2), 127-130. doi:10.1016/j.antiviral.2004.05.006

Grabherr, M. G., Haas, B. J., Yassour, M., Levin, J. Z., Thompson, D. A., Amit, I., . . Regev, A. (2011). Trinity: reconstructing a full-length transcriptome without a genome from RNA-Seq data. Nat Biotechnol, 29(7), 644-652. doi:10.1038/nbt.1883

Gremme, G., Steinbiss, S., \& Kurtz, S. (2013). GenomeTools: a comprehensive software library for efficient processing of structured genome annotations. IEEE/ACM Trans Comput Biol Bioinform, 10(3), 645-656. doi:10.1109/TCBB.2013.68

Haas, B. J., Delcher, A. L., Wortman, J. R., \& Salzberg, S. L. (2004). DAGchainer: a tool for mining segmental genome duplications and synteny. Bioinformatics, 20(18), 3643-3646. doi:10.1093/bioinformatics/bth397

Haas, B. J., Salzberg, S. L., Zhu, W., Pertea, M., Allen, J. E., Orvis, J., . . Wortman, J. R. (2008). Automated eukaryotic gene structure annotation using EVidenceModeler and the Program to Assemble Spliced Alignments. Genome Biol, 9(1), R7-R7. doi:10.1186/gb-2008-9-1-r7

Hage, H., Miyauchi, S., Viragh, M., Drula, E., Min, B., Chaduli, D., .. Rosso, M. N. (2021). Gene family expansions and transcriptome signatures uncover fungal adaptations to wood decay. Environ Microbiol. doi:10.1111/1462-2920.15423

Hibbett, D. S., \& Thorn, R. G. (2001). Basidiomycota: Homobasidiomycetes. In D. J. McLaughlin, E. G. McLaughlin, \& P. A. Lemke (Eds.), Systematics and Evolution (pp. 121-168). Berlin, Heidelberg: Springer Berlin Heidelberg. 
Hoff, K. J., Lange, S., Lomsadze, A., Borodovsky, M., \& Stanke, M. (2015). BRAKER1: Unsupervised RNA-Seq-Based Genome Annotation with GeneMark-ET and AUGUSTUS. Bioinformatics, 32(5), 767-769. doi:10.1093/bioinformatics/btv661

Holonec, L., Ranga, F., Crainic, D., Truța, A., \& Socaciu, C. (2012). Evaluation of Betulin and Betulinic Acid Content in Birch Bark from Different Forestry Areas of Western Carpathians. Notulae Botanicae Horti Agrobotanici Cluj-Napoca, 40. doi:10.15835/nbha4027967

Hu, Z., Guo, N., Wang, Z., Liu, Y., Wang, Y., Ding, W., . . Yan, X. (2013). Development and validation of an LC-ESI/MS/MS method with precolumn derivatization for the determination of betulin in rat plasma. J Chromatogr B Analyt Technol Biomed Life Sci, 939, 38-44. doi:10.1016/j.jchromb.2013.09.005

Ide, M., Ichinose, H., \& Wariishi, H. (2012). Molecular identification and functional characterization of cytochrome P450 monooxygenases from the brown-rot basidiomycete Postia placenta. Archives of Microbiology, 194(4), 243-253. doi:10.1007/s00203-011-0753-2

Kang, S., Lebrun, M. H., Farrall, L., \& Valent, B. (2001). Gain of virulence caused by insertion of a Pot3 transposon in a Magnaporthe grisea avirulence gene. Mol Plant Microbe Interact, 14(5), 671-674. doi:10.1094/MPMI.2001.14.5.671

Khelil, R., Jardé, E., Cabello-Hurtado, F., Ould-el-Hadj Khelil, A., \& Esnault, M.-A. (2016). Structure and composition of the wax of the date palm, Phoenix dactylifera L., from the septentrional Sahara. Scientia Horticulturae, 201, 238-246. doi:https://doi.org/10.1016/j.scienta.2016.02.012

Khouloud Barakat, M. S. (2016). Bioactive Betulin produced by marine Paecilomyces WE3-F. J Appl Pharm Sci(Volume: 6, Issue: 3), 034-040. Retrieved from http://japsonline.com/abstract.php?article id=1799

Kim, D., Pertea, G., Trapnell, C., Pimentel, H., Kelley, R., \& Salzberg, S. L. (2013). TopHat2: accurate alignment of transcriptomes in the presence of insertions, deletions and gene fusions. Genome Biol, 14(4), R36. doi:10.1186/gb-2013-14-4-r36

Koolen, H. H. F., Soares, E. R., Silva, F. M. A. d., Souza, A. Q. L. d., Rodrigues Filho, E., \& Souza, A. D. L. d. (2012). Triterpenes and flavonoids from the roots of Mauritia flexuosa. Rev Bras Farmacogn, 22, 189-192. Retrieved from http://www.scielo.br/scielo.php?script=sci_arttext\&pid=S0102695X2012000100028\&nrm=iso

Król, S. K., Kiełbus, M., Rivero-Müller, A., \& Stepulak, A. (2015). Comprehensive Review on Betulin as a Potent Anticancer Agent. BioMed Research International, 2015, 11. doi:10.1155/2015/584189

Kumar, S., Stecher, G., Suleski, M., \& Hedges, S. B. (2017). TimeTree: A Resource for Timelines, Timetrees, and Divergence Times. Mol Biol Evol, 34(7), 1812-1819. doi:10.1093/molbev/msx116

Lepesheva, G. I., Hargrove, T. Y., Kleshchenko, Y., Nes, W. D., Villalta, F., \& Waterman, M. R. (2008). CYP51: A major drug target in the cytochrome P450 superfamily. Lipids, 43(12), 1117-1125. doi:10.1007/s11745-008-3225-y

Liu, Y., Wu, Y., Zhang, Y., Yang, X., Yang, E., Xu, H., . . Yan, J. (2019). Lignin degradation potential and draft genome sequence of Trametes trogii S0301. Biotechnol Biofuels, 12, 256. doi:10.1186/s13068-019-1596-3

Lodhi, M., Ye, G.-N., Weeden, N., \& I. Reisch, B. (1994). A simple and efficient method for DNA extraction from grapevine cultivars andVitis species. Plant Mol Biol Rep, 12(1), 6-13. doi:10.1007/BF02668658

Love, M. I., Huber, W., \& Anders, S. (2014). Moderated estimation of fold change and dispersion for RNA-seq data with DESeq2. Genome Biol, 15(12), 550. doi:10.1186/s13059-014-0550-8 
Ma, L., Chen, H., Dong, P., \& Lu, X. (2013). Anti-inflammatory and anticancer activities of extracts and compounds from the mushroom Inonotus obliquus. Food Chem, 139(1-4), 503-508. doi:10.1016/j.foodchem.2013.01.030

Matheny, P. B., Wang, Z., Binder, M., Curtis, J. M., Lim, Y. W., Nilsson, R. H., . . Hibbett, D. S. (2007). Contributions of rpb2 and tef1 to the phylogeny of mushrooms and allies (Basidiomycota, Fungi). Mol Phylogenet Evol, 43(2), 430-451. doi:10.1016/j.ympev.2006.08.024

Miettinen, K., Pollier, J., Buyst, D., Arendt, P., Csuk, R., Sommerwerk, S., . . Goossens, A. (2017). The ancient CYP716 family is a major contributor to the diversification of eudicot triterpenoid biosynthesis. Nat Commun, 8, 14153.

Nagajyothi, P. C., Sreekanth, T. V., Lee, J. I., \& Lee, K. D. (2014). Mycosynthesis: antibacterial, antioxidant and antiproliferative activities of silver nanoparticles synthesized from Inonotus obliquus (Chaga mushroom) extract. J Photochem Photobiol B, 130, 299-304. doi:10.1016/j.jphotobiol.2013.11.022

Navarro, D., Chaduli, D., Taussac, S., Lesage-Meessen, L., Grisel, S., Haon, M., . . Favel, A. (2021). Large-scale phenotyping of 1,000 fungal strains for the degradation of non-natural, industrial compounds. Communications Biology, 4(1), 871. doi:10.1038/s42003-021-02401w

P. Kovalenko, L., Shipaeva, E., V. Balakshin, V., A. Presnova, G., N. Chistyakov, A., Klodt, P., .. . Durnev, A. (2009). Antiallergenic activity of birch bark dry extract with at least $70 \%$ betulin content. Pharm Chem J, 43, 110-114. doi:10.1007/s11094-009-0242-y

Panchy, N., Lehti-Shiu, M., \& Shiu, S.-H. (2016). Evolution of Gene Duplication in Plants. Plant Physiology, 171(4), 2294-2316. doi:10.1104/pp.16.00523

Quevillon, E., Silventoinen, V., Pillai, S., Harte, N., Mulder, N., Apweiler, R., \& Lopez, R. (2005). InterProScan: protein domains identifier. Nucleic Acids Res, 33(Web Server issue), W116120. doi:10.1093/nar/gki442

Rice, P., Longden, I., \& Bleasby, A. (2000). EMBOSS: the European Molecular Biology Open Software Suite. Trends Genet, 16(6), 276-277. Retrieved from https://www.ncbi.nlm.nih.gov/pubmed/10827456

Ryvarden, L., \& Gilbertson, R. L. (1993). European polypores. 1 : Abortiporus-Lindtneria: Oslo : Fungiflora.

Safronov, O., Alonso-Serra, J., Lim, K. J., Fraser-Miller, S. J., Blokhina, O. B., Campilho, A., . . . Salojarvi, J. (2019). Tissue-specific study across the stem reveals the chemistry and transcriptome dynamics of birch bark. New Phytol, 222(4), 1816-1831. doi:10.1111/nph.15725

Salin, O., Alakurtti, S., Pohjala, L., Siiskonen, A., Maass, V., Maass, M., ... Vuorela, P. (2010). Inhibitory effect of the natural product betulin and its derivatives against the intracellular bacterium Chlamydia pneumoniae. Biochem Pharmacol, 80(8), 1141-1151. doi:10.1016/j.bcp.2010.06.051

Salojärvi, J., Smolander, O.-P., Nieminen, K., Rajaraman, S., Safronov, O., Safdari, P., . . . Kangasjärvi, J. (2017). Genome sequencing and population genomic analyses provide insights into the adaptive landscape of silver birch. Nat Genet, 49, 904. doi:10.1038/ng.3862

Schlapfer, P., Zhang, P., Wang, C., Kim, T., Banf, M., Chae, L., . . Rhee, S. Y. (2017). Genome-Wide Prediction of Metabolic Enzymes, Pathways, and Gene Clusters in Plants. Plant Physiol, 173(4), 2041-2059. doi:10.1104/pp.16.01942

Sezutsu, H., Le Goff, G., \& Feyereisen, R. (2013). Origins of P450 diversity. Philosophical transactions of the Royal Society of London. Series B, Biological sciences, 368(1612), 20120428-20120428. doi:10.1098/rstb.2012.0428 
Shai, L. J., McGaw, L. J., Aderogba, M. A., Mdee, L. K., \& Eloff, J. N. (2008). Four pentacyclic triterpenoids with antifungal and antibacterial activity from Curtisia dentata (Burm.f) C.A. Sm. leaves. J Ethnopharmacol, 119(2), 238-244. doi:10.1016/j.jep.2008.06.036

Siddiqui, S. A., Rahman, A., Rahman, M. O., Akbar, M. A., Ali, M. A., Al-Hemaid, F. M. A., ... Farah, M. A. (2019). A novel triterpenoid 16-hydroxy betulinic acid isolated from Mikania cordata attributes multi-faced pharmacological activities. Saudi J Biol Sci, 26(3), 554-562. doi:10.1016/j.sjbs.2018.03.002

Šiman, P., Filipová, A., Tichá, A., Niang, M., Bezrouk, A., \& Havelek, R. (2016). Effective Method of Purification of Betulin from Birch Bark: The Importance of Its Purity for Scientific and Medicinal Use. PLOS ONE, 11(5), e0154933. doi:10.1371/journal.pone.0154933

Slater, G. S. C., \& Birney, E. (2005). Automated generation of heuristics for biological sequence comparison. BMC Bioinformatics, 6(1), 31. doi:10.1186/1471-2105-6-31

Soneson, C., Love, M. I., \& Robinson, M. D. (2015). Differential analyses for RNA-seq: transcriptlevel estimates improve gene-level inferences. F1000Res, 4, 1521. doi:10.12688/f1000research.7563.2

Song, F. Q., Liu, Y., Kong, X. S., Chang, W., \& Song, G. (2013). Progress on understanding the anticancer mechanisms of medicinal mushroom: inonotus obliquus. Asian Pac J Cancer Prev, 14(3), 1571-1578. Retrieved from https://www.ncbi.nlm.nih.gov/pubmed/23679238

Stamatakis, A. (2014). RAxML version 8: a tool for phylogenetic analysis and post-analysis of large phylogenies. Bioinformatics, 30(9), 1312-1313. doi:10.1093/bioinformatics/btu033

Stanke, M., \& Morgenstern, B. (2005). AUGUSTUS: a web server for gene prediction in eukaryotes that allows user-defined constraints. Nuc acids res, 33. doi:10.1093/nar/gki458

Suyama, M., Torrents, D., \& Bork, P. (2006). PAL2NAL: robust conversion of protein sequence alignments into the corresponding codon alignments. Nucleic Acids Res, 34(Web Server issue), W609-612. doi:10.1093/nar/gkl315

Tang, H., Krishnakumar, V., Bidwell, S., Rosen, B., Chan, A., Zhou, S., . . Town, C. D. (2014). An improved genome release (version Mt4.0) for the model legume Medicago truncatula. BMC Genomics, 15, 312. doi:10.1186/1471-2164-15-312

The French-Italian Public Consortium for Grapevine Genome, C., Jaillon, O., Aury, J.-M., Noel, B., Policriti, A., Clepet, C., . . . Wincker, P. (2007). The grapevine genome sequence suggests ancestral hexaploidization in major angiosperm phyla. Nature, 449, 463. doi:10.1038/nature06148

https://www.nature.com/articles/nature06148\#supplementary-information

Waterhouse, R. M., Seppey, M., Simão, F. A., Manni, M., loannidis, P., Klioutchnikov, G., . . . Zdobnov, E. M. J. M. B. E. (2017). BUSCO applications from quality assessments to gene prediction and phylogenomics. doi:10.1093/molbev/msx319

Wu, J., Niu, Y., Bakur, A., Li, H., \& Chen, Q. (2017). Cell-Free Production of Pentacyclic Triterpenoid Compound Betulinic Acid from Betulin by the Engineered Saccharomyces cerevisiae. Molecules, 22(7). doi:10.3390/molecules22071075

Yan, Z. F., Yang, Y., Tian, F. H., Mao, X. X., Li, Y., \& Li, C. T. (2014). Inhibitory and Acceleratory Effects of Inonotus obliquus on Tyrosinase Activity and Melanin Formation in B16 Melanoma Cells. Evid Based Complement Alternat Med, 2014, 259836. doi:10.1155/2014/259836

Yap, H.-Y. Y., Chooi, Y.-H., Firdaus-Raih, M., Fung, S.-Y., Ng, S.-T., Tan, C.-S., \& Tan, N.-H. (2014). The genome of the Tiger Milk mushroom, Lignosus rhinocerotis, provides insights into the genetic basis of its medicinal properties. BMC Genomics, 15(1), 635. doi:10.1186/14712164-15-635 
862

863

864

865

866

867

868

869

870

871

872

873

874

875

876

877

878

879

880

881

882

883

884

885

886

887

888

889

890

891

892

893

894

895

Yin, Y., Cui, Y., \& Ding, H. (2008). Optimization of betulin extraction process from Inonotus Obliquus with pulsed electric fields. Innov Food Sci Emerg Technol, 9(3), 306-310. doi:https://doi.org/10.1016/j.ifset.2007.07.010

Yu, F., Song, J., Liang, J., Wang, S., \& Lu, J. (2020). Whole genome sequencing and genome annotation of the wild edible mushroom, Russula griseocarnosa. Genomics, 112(1), 603614. doi:https://doi.org/10.1016/j.ygeno.2019.04.012

Zhang, H., Yohe, T., Huang, L., Entwistle, S., Wu, P., Yang, Z., ... Yin, Y. (2018). dbCAN2: a meta server for automated carbohydrate-active enzyme annotation. Nucleic Acids Res, 46(W1), W95-W101. doi:10.1093/nar/gky418

Zhang, H. J., Tan, G. T., Hoang, V. D., Hung, N. V., Cuong, N. M., Soejarto, D. D., . . Fong, H. H. (2003). Natural anti-HIV agents. Part IV. Anti-HIV constituents from Vatica cinerea. J Nat Prod, 66(2), 263-268. doi:10.1021/np020379y

Zhao, G., Yan, W., \& Cao, D. (2007). Simultaneous determination of betulin and betulinic acid in white birch bark using RP-HPLC. J Pharm Biomed Anal, 43(3), 959-962. doi:10.1016/j.jpba.2006.09.026

Zhao, R.-L., Li, G.-J., Sánchez-Ramírez, S., Stata, M., Yang, Z.-L., Wu, G., . . Hyde, K. D. (2017). A sixgene phylogenetic overview of Basidiomycota and allied phyla with estimated divergence times of higher taxa and a phyloproteomics perspective. Fungal Diversity, 84(1), 43-74. doi:10.1007/s13225-017-0381-5

Zhao, S., Park, C. H., Li, X., Kim, Y. B., Yang, J., Sung, G. B., . . Park, S. U. (2015). Accumulation of Rutin and Betulinic Acid and Expression of Phenylpropanoid and Triterpenoid Biosynthetic Genes in Mulberry (Morus alba L.). J Agric Food Chem, 63(38), 8622-8630. doi:10.1021/acs.jafc.5b03221

Zhou, C., Li, J., Li, C., \& Zhang, Y. (2016). Improvement of betulinic acid biosynthesis in yeast employing multiple strategies. BMC Biotechnology, 16(1), 59. doi:10.1186/s12896-0160290-9

Zhu, F., Moural, T. W., Shah, K., \& Palli, S. R. (2013). Integrated analysis of cytochrome P450 gene superfamily in the red flour beetle, Tribolium castaneum. BMC Genomics, 14(1), 174. doi:10.1186/1471-2164-14-174

Zuo, M., Gao, M. J., Liu, Z., Cai, L., \& Duan, G. L. (2005). p-Toluenesulfonyl isocyanate as a novel derivatization reagent to enhance the electrospray ionization and its application in the determination of two stereo isomers of 3-hydroxyl-7-methyl-norethynodrel in plasma. $J$ Chromatogr B Analyt Technol Biomed Life Sci, 814(2), 331-337. doi:10.1016/j.jchromb.2004.10.054 


\section{Figures:}

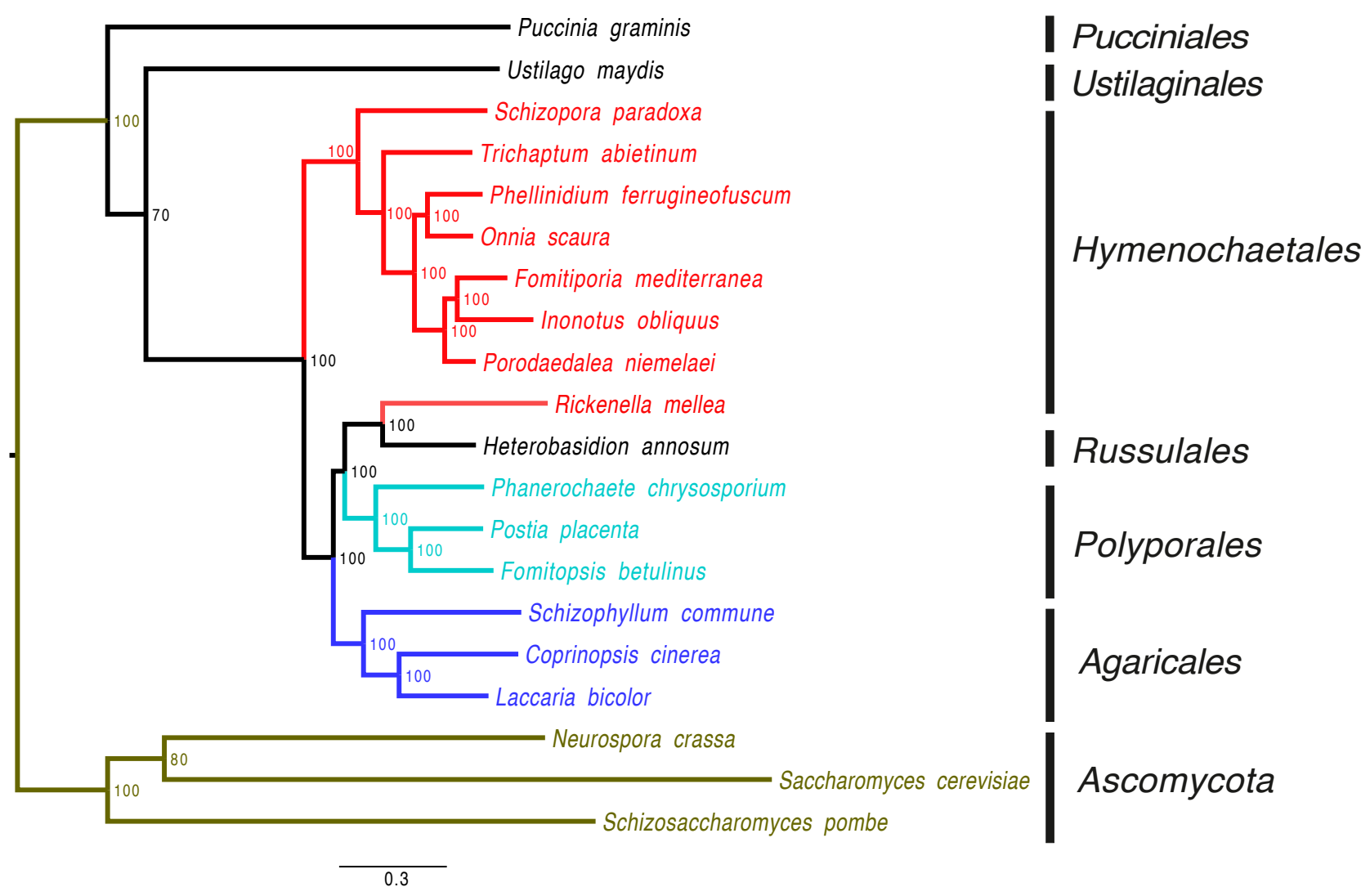

Figure 1. Phylogenetic tree of three Ascomycetes and 17 Basidiomycetes species. Ascomycetes are highlighted with green colour and grouped by Phylum. Basidiomycetes species are grouped by taxonomic order. Bootstrap values are plotted next to the nodes illustrating the level of the confidence of the split, and the phylogenetic tree was rooted to Ascomycetes clade. 
S. paradoxa vs S. paradoxa

P. niemelaei vs P. niemelaei

I. obliquus vs S. paradoxa

I. obliquus vs $P$. niemelaei

I. obliquus vs F. mediterranea

F. mediterranea vs F. mediterranea

\section{F. betulina vs F. betulina}

I. obliquus vs F. betulina

Figure 2. Density plots of the number of synonymous (Ks) substitutions in syntelogs identified from syntenic alignments of I. obliquus, F. mediterranea, P. niemelaei, and S. paradoxa. X-axis is displayed as $\log 10$ of synonymous substitutions per synonymous site (Ks). 


\section{Syntenic Tandem}
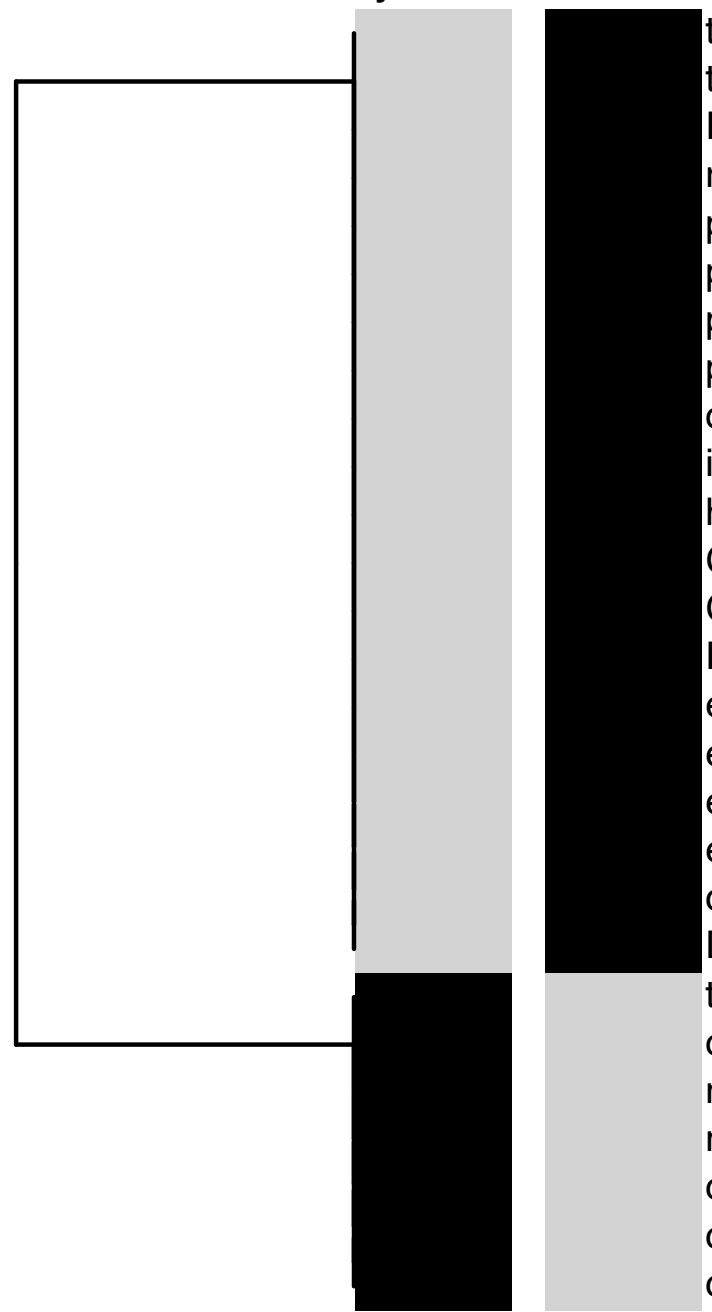

transition metal ion binding

NA

tetrapyrrole binding

Enriched GOs

RNA-DNA hybrid ribonuclease activity

ribonuclease activity

protein-containing complex binding

protein phosphorylation

protein kinase activity

phosphorylation

oxidoreductase activity_GO:0016705

iron ion binding

heme binding

G-protein beta/gamma-subunit complex binding

$G$ protein-coupled receptor signaling pathway

FMN binding

extracellular space

endoribonuclease activity_GO:0016891

endoribonuclease activity

endonuclease activity_GO:0016893

carbohydrate biosynthetic process

DNA integration

terpene synthase activity

organelle fission

nuclear division

mitotic chromosome condensation

condensin complex

carbon-oxygen lyase activity, acting on phosphates

chromosome condensation

Figure 3. Gene ontology (GO) enrichment of genes identified in syntenic and tandemly duplicated regions in I. obliquus and four other species. Separate heatmaps are shown for the syntenic and tandemly duplicated regions, black color shows significantly enriched GOs and grey illustrates nonsignificant enrichments (NS). GOs are clustered using the Euclidean distance and hierarchical clustering (method: complete). 


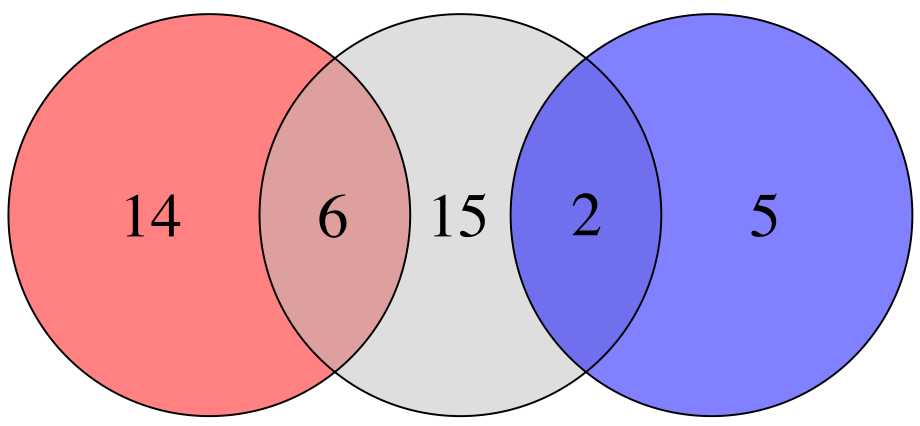

\section{Tandem Syntenic}

p.value: $6.1094 \mathrm{e}-11$

Figure 4: Venn diagram of $\mathrm{GO}$ enrichment analysis for expanded gene families and tandemly duplicated genes from $I$. obliquus genome. Each category is highlighted and labeled by specific color. P-value was calculated with Fisher exact test assessing the the statistical significance of the overlap. Tandem: tandemly duplication genes; Expanded: expanded gene families; syntenic: syntenic self-self alignment of $I$. obliquus resulting in the set of genes originating from whole genome duplication event. 


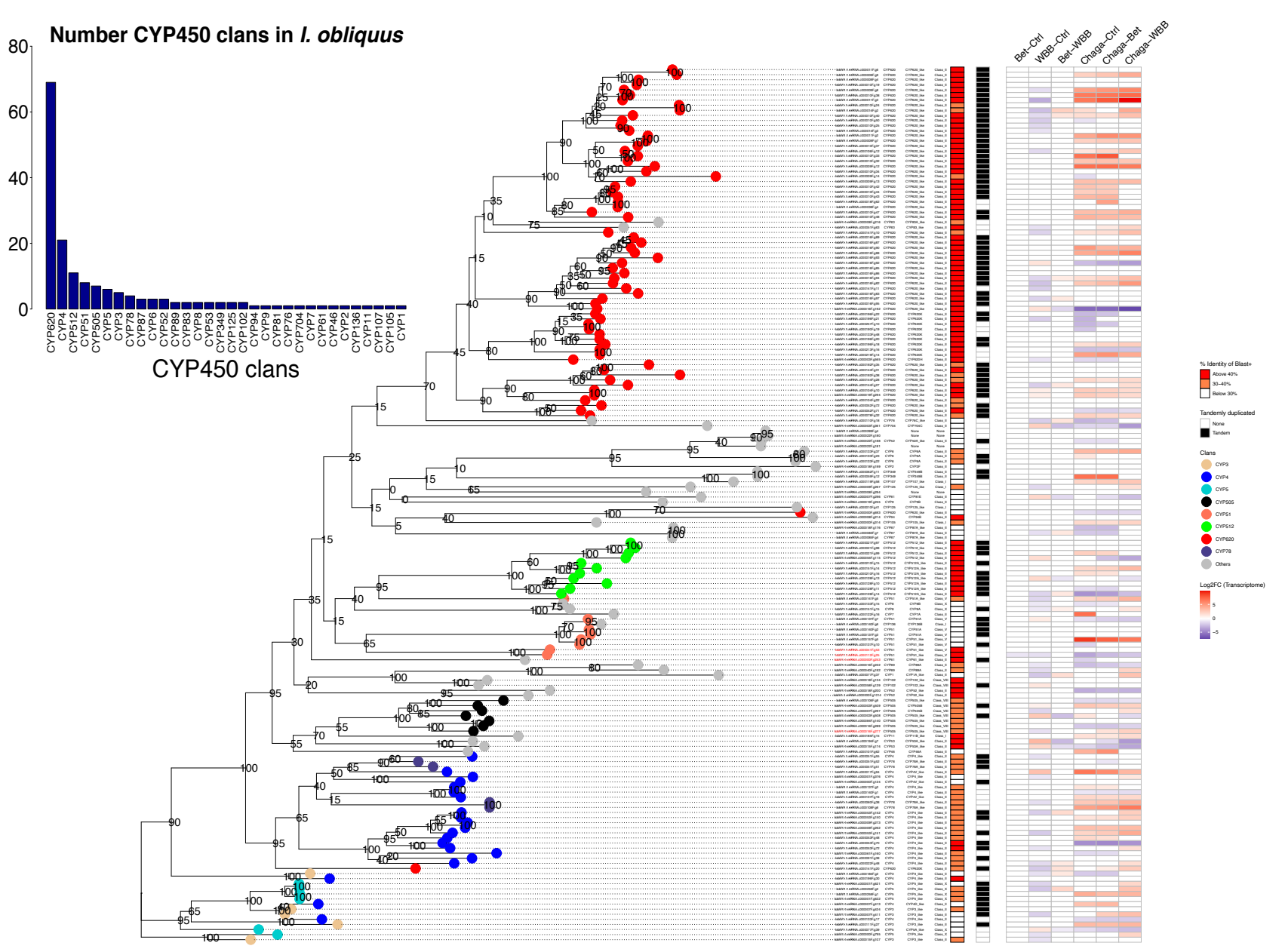

Figure 5. A gene tree of CYP450s predicted in I. obliquus. The clades are highlighted according to the major clan of the enzyme and tandemly duplicated genes are indicated with black squares; the color scheme is shown in the color key to the right of the plots. The branches are labeled by gene ID, cytochrome P450 clans, family, and class. The heatmaps illustrate BLAST similarities (red), tandemly duplicated (black/white), and differential expression of the genes. Barplot in the inset shows the number of CYP450 clans in I. obliquus. 
A

\section{Chaga strains}

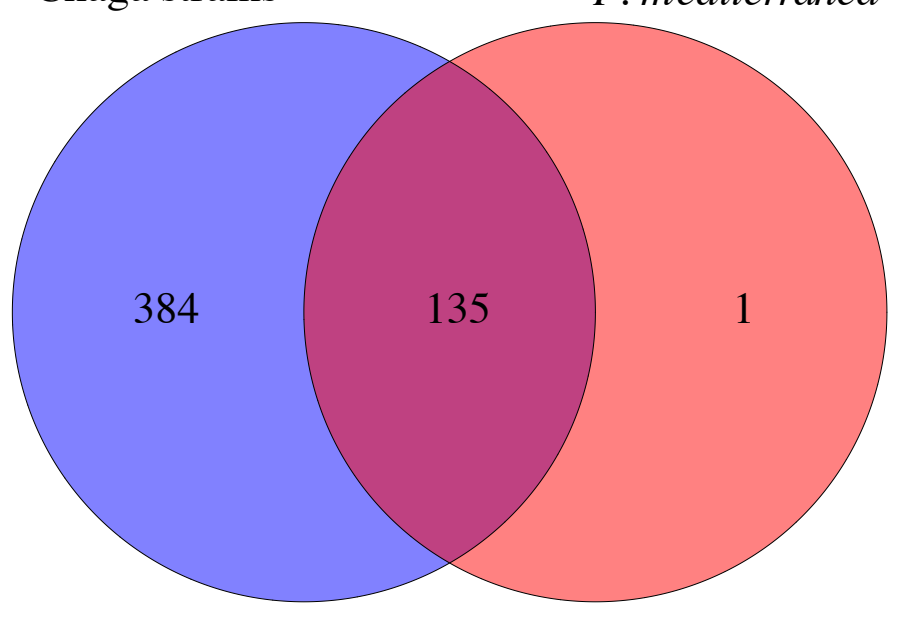

B

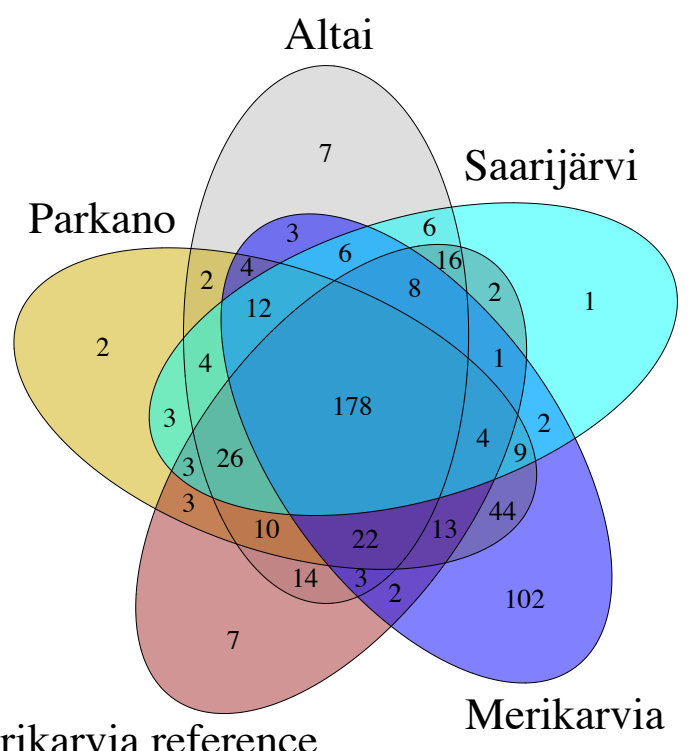

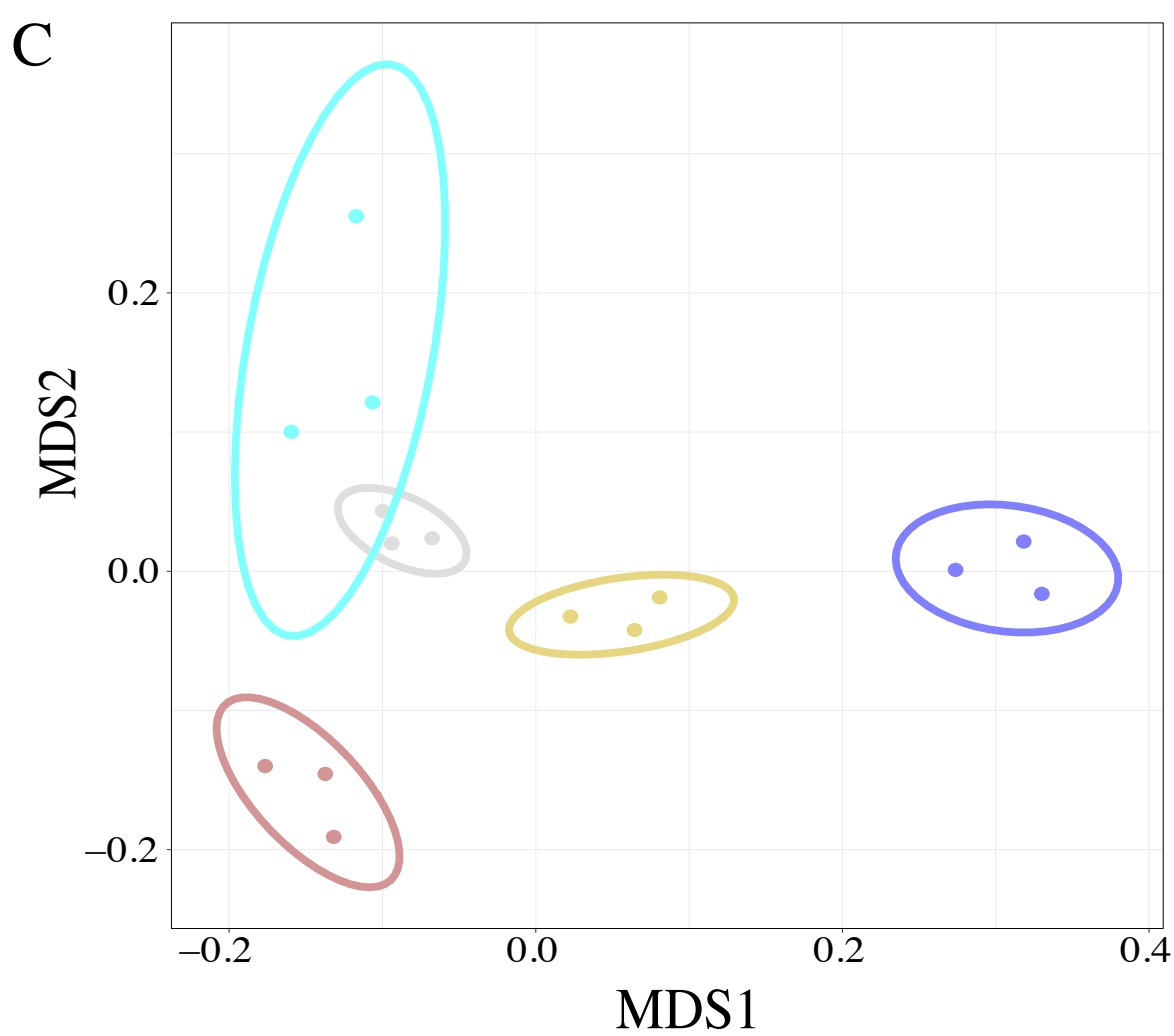

Native location

Altai

Merikarvia reference

Merikarvia

Parkano

Saarijärvi

Figure 6: Venn diagrams of UPLC-MS mass spectrum for metabolomic fingerprints. A) Pooled mass spectrums from five strains of $I$. obliquus and one $F$. mediterranea, B) mass spectrums of five strains of $I$. obliquus, C) multidimensional scaling (MDS) plot of metabolomics abundant of five strains of $I$. obliquus. Panels B and C use the same color coding, explained in panel Clegend. . 


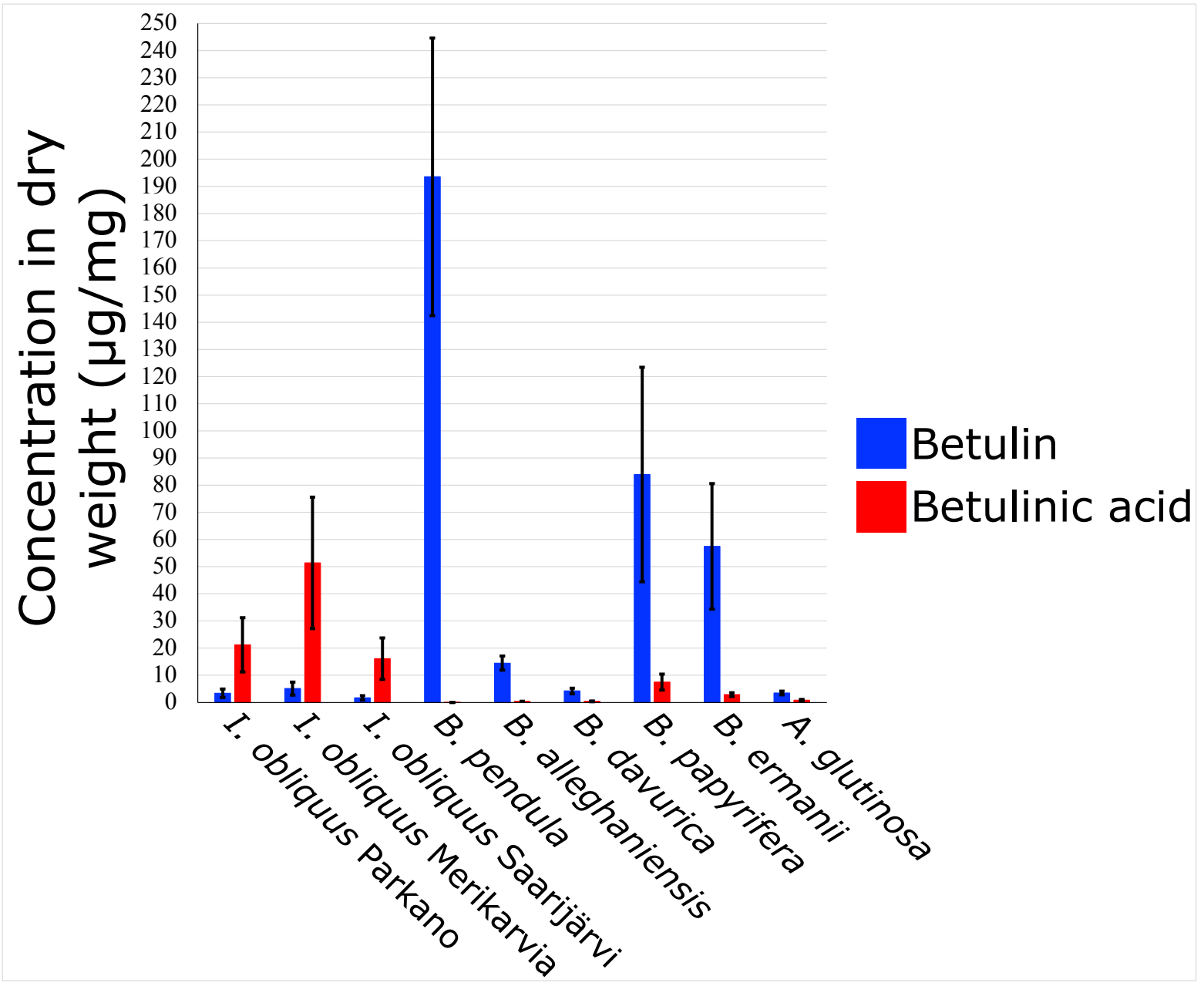

Figure 7: Concentration of betulin and betulinic acid in three strains of chaga and six betula species by HPLC-MS. Betulin and betulinic acid are highlighted in red and blue. 


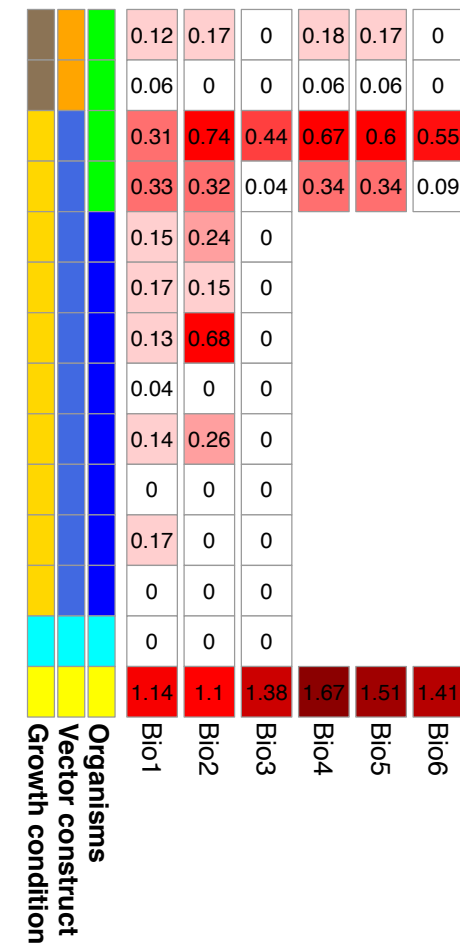

pRS424::LUS-CYP716 Yeast extract

pRS424::LUS-CYP716 Culture media

pRS424::CYP716 Yeast extract

Betulin concentration $\mu \mathrm{g} / \mathrm{mL}$

pRS424::CYP716 Culture media

pRS424::CYP716 Yeast extract (c000000F_g253_1)

pRS424::CYP716 Culture media (C000000F_g253_1)

pRS424::CYP716 Yeast extract (c000016F_g277)

pRS424::CYP716 Culture media (c000016F_g277)

pRS424::CYP716 Yeast extract (c000041F_g53)

Growth condition

pRS424::CYP716 Culture media (c000041F_g53)

pRS424::CYP716 Yeast extract (c000112F_g25)

pRS424::CYP716 Culture media (c000112F_g25)

Yeast CDD6 strain (Yeast extract/Culture media)

1.5

1

0.5

0

Standard Betulin (Sigma-Aldrich 98\%)

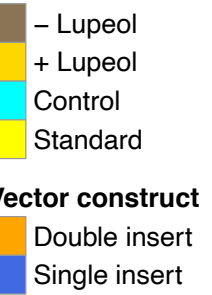

Organisms

Birch

Chaga

Figure 8: Heatmap illustrating the amount of betulin synthesized in transgenic yeast. The columns illustrate the biological replicates, and rows the vector constructs for lupeol synthase and CYP716 genes. The biological source for lupeol synthase was birch (green, B. pendula), and the biological sources for CYP716 enzymes were from birch and chaga mushroom (dark blue, l. obliquus). Vector constructs were divided to double insert (orange, lupeol synthase and CYP716 from birch in a single vector), and single inserts (royal blue, only CYP716 from birch and chaga). The color palette of the heatmap illustrates the concentrations $(\mu \mathrm{g} / \mathrm{mL}$ ) of betulin found in yeast cell (Yeast extract) and yeast growth media (Culture media), with white color assigned to minimum and dark red to maximum concentration of betulin. The second heatmap illustrates the growth conditions: brown (- Lupeol) is yeast growth media without standard lupeol (precursor for CYP716 gene), gold (+ Lupeol) yeast growth media with standard lupeol, and cyan CDD6 yeast strain used as control, and finally yellow is lupeol standard ( $98 \%$ purity). 


\section{Monooxygenase P450 (Nucleotides)}

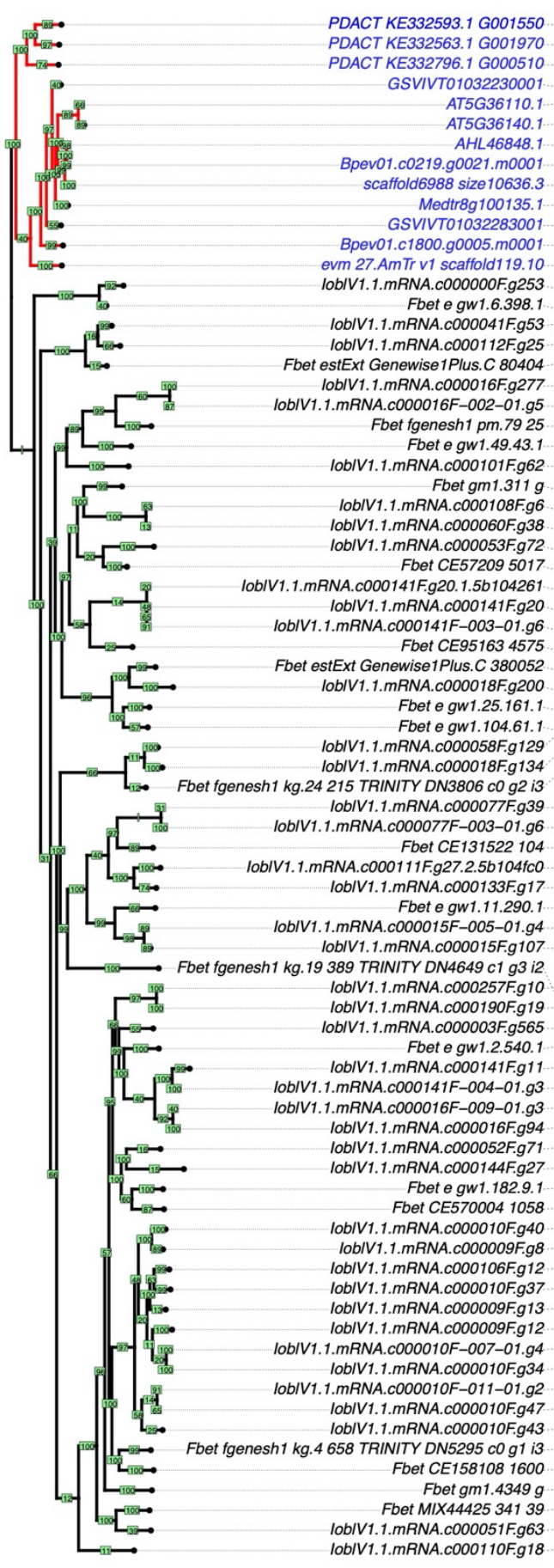

Monooxygenase P450 (Amino acids)

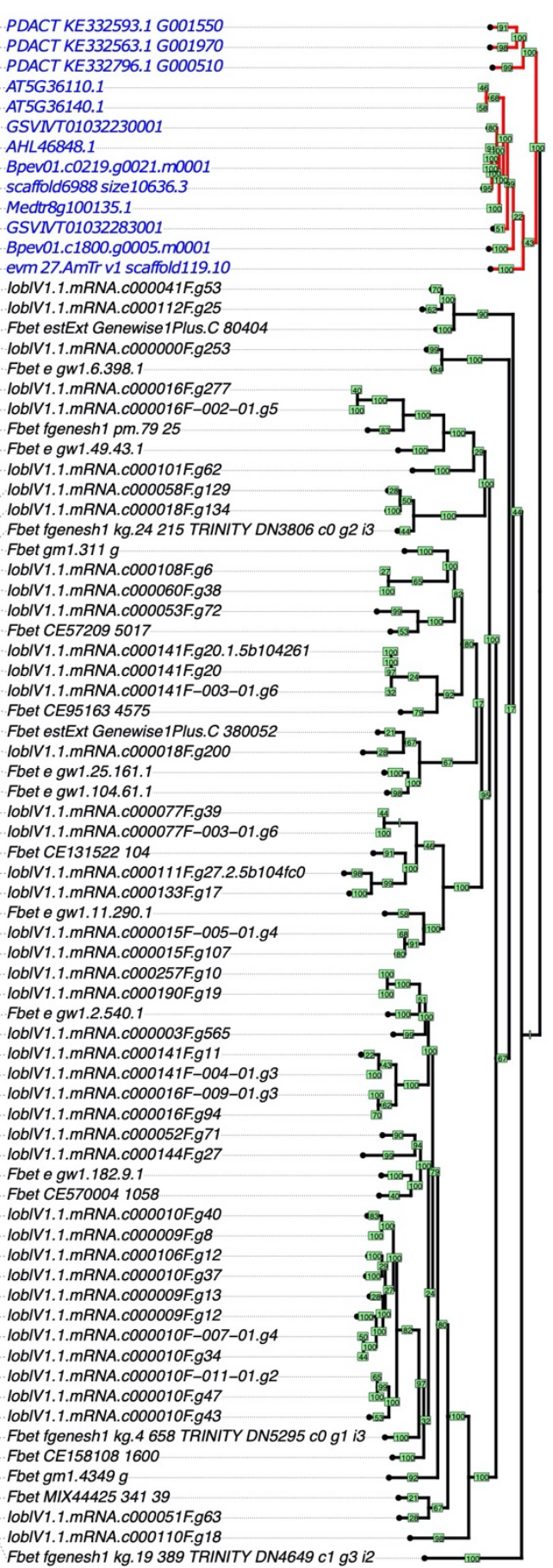

Figure 9: Phylogenetic reconstruction of 70 monooxygenase enzymes from I. obliquus, F. betulina, and 8 plant species. Trees are labeled according to the type of the sequence used in multiple sequence alignment (amino acid or nucleotide) and rooted to plant species. Cophylo (from phytools) function is used in order to rotate the branches to match the tips and the labels. Bootstrap values (green rectangular) illustrate the level of the confidence, and plant species are highlighted in blue. 


\section{Mevalonate pathway}

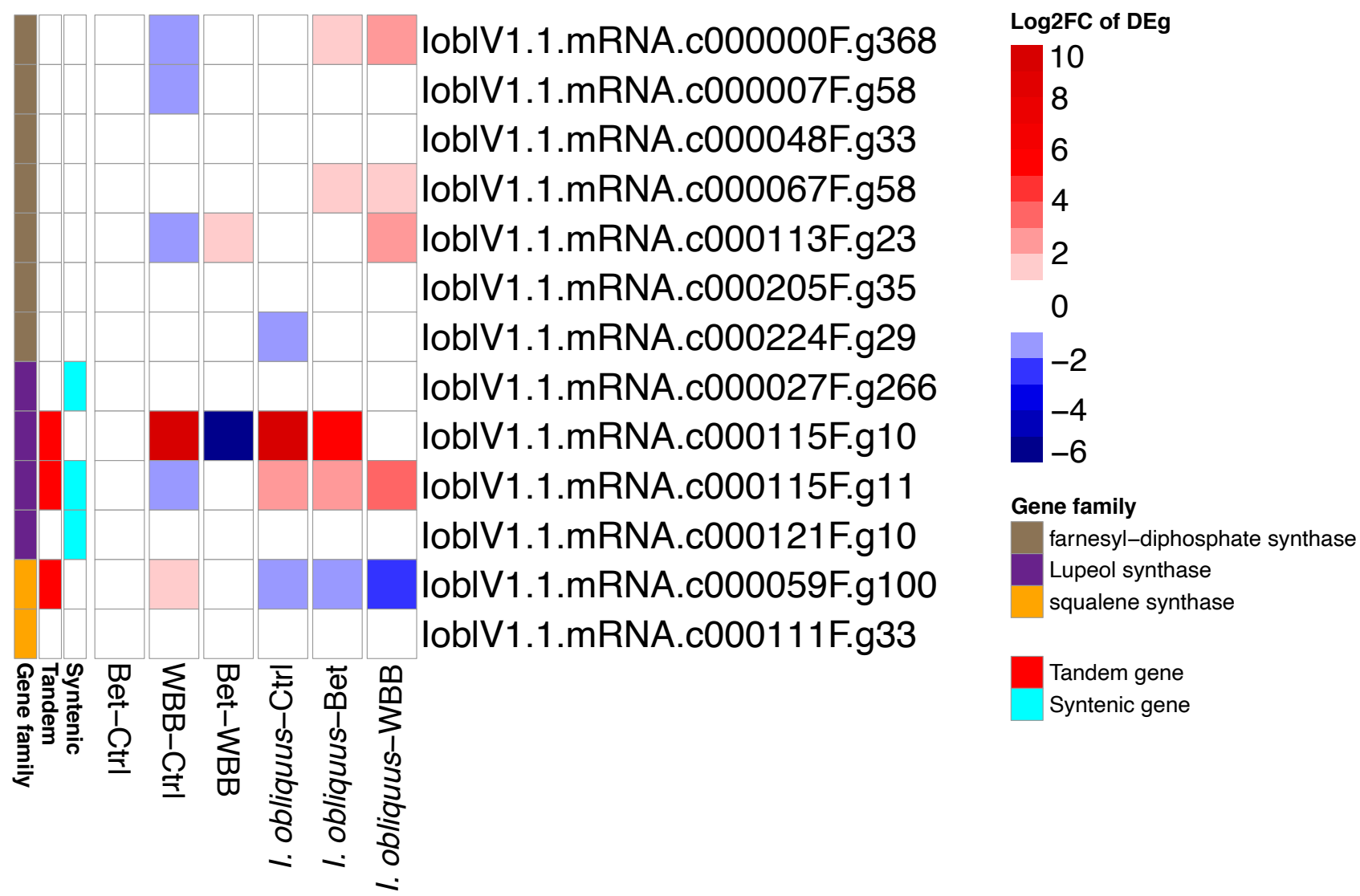

Figure 10: Heatmap of differentially expressed (DE) genes annotated: A) CYP450 monooxygenase enzymes, and B) key enzymes in mevalonate pathways (MVA). The larger heatmap illustrates the $\log _{2}$ fold-changes $\left(\log _{2} \mathrm{FC}\right)$ of $\mathrm{DE}$ genes, the color is proportional to differential expression. The smaller heatmap illustratres the duplication origins of the gene, either syntenic originating from whole genome duplication or tandem originating from segmental duplication. Genes are clustered using the Euclidean distance and hierarchical clustering (method: complete). Gene ID highlighted in blue was selected for cloning. 
bioRxiv preprint doi: https://doi.org/10.1101/2021.11.28.470225; this version posted November 28, 2021. The copyright holder for this preprint (which was not certified by peer review) is the author/funder, who has granted bioRxiv a license to display the preprint in perpetuity. It is made available under aCC-BY-NC-ND 4.0 International license.

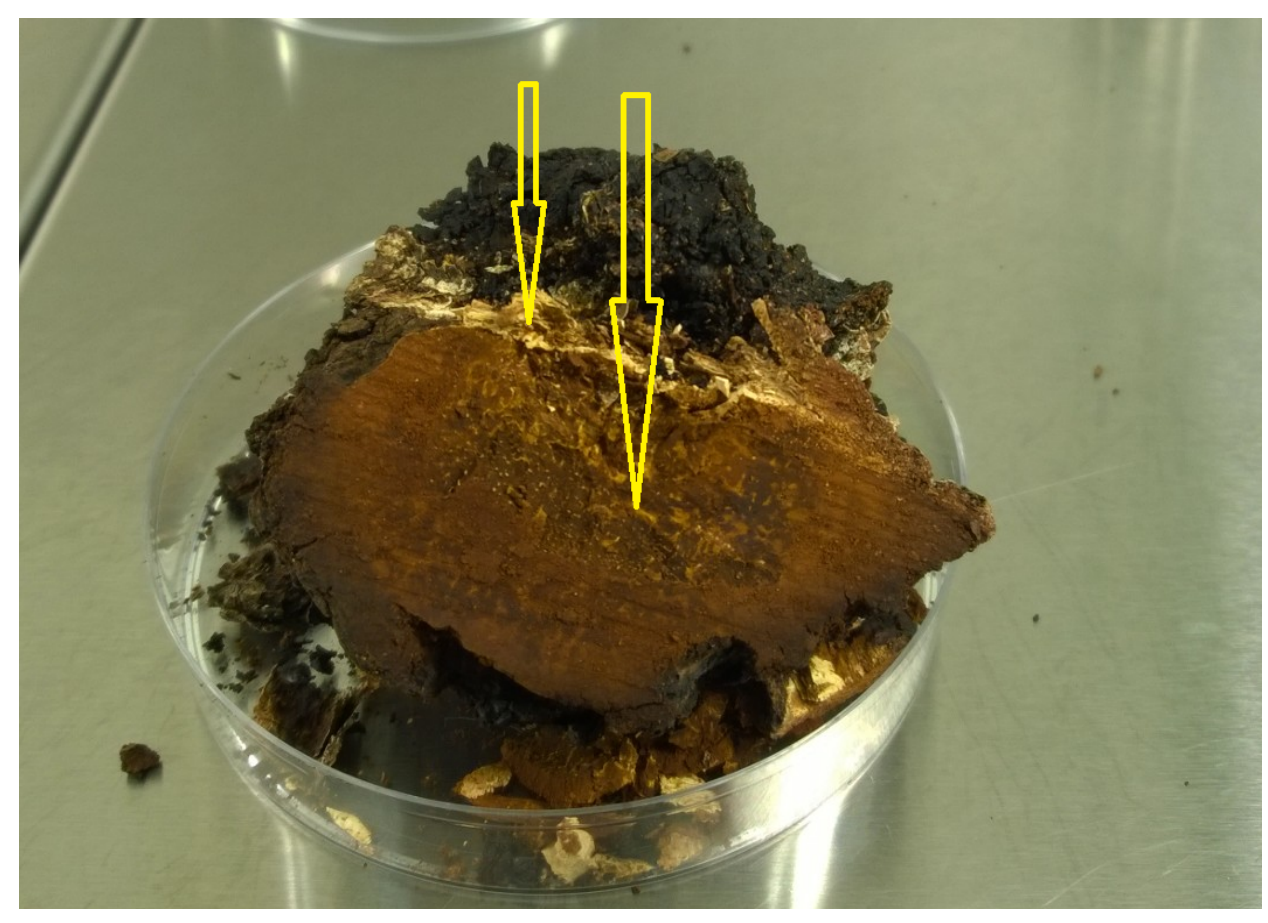

Supp. Fig 1: Fragment of Inonotus obliquus conk collected from Merikarvia, Finland [N62.00', E24.74 ${ }^{\circ}$ ]. Yellow arrows point at sites where samples were collected for inoculation of culture media. There might be contamination of birch bark at the site where the smaller arrow is pointing. The larger arrow points to the center of the conk where more pure sample was collected. 


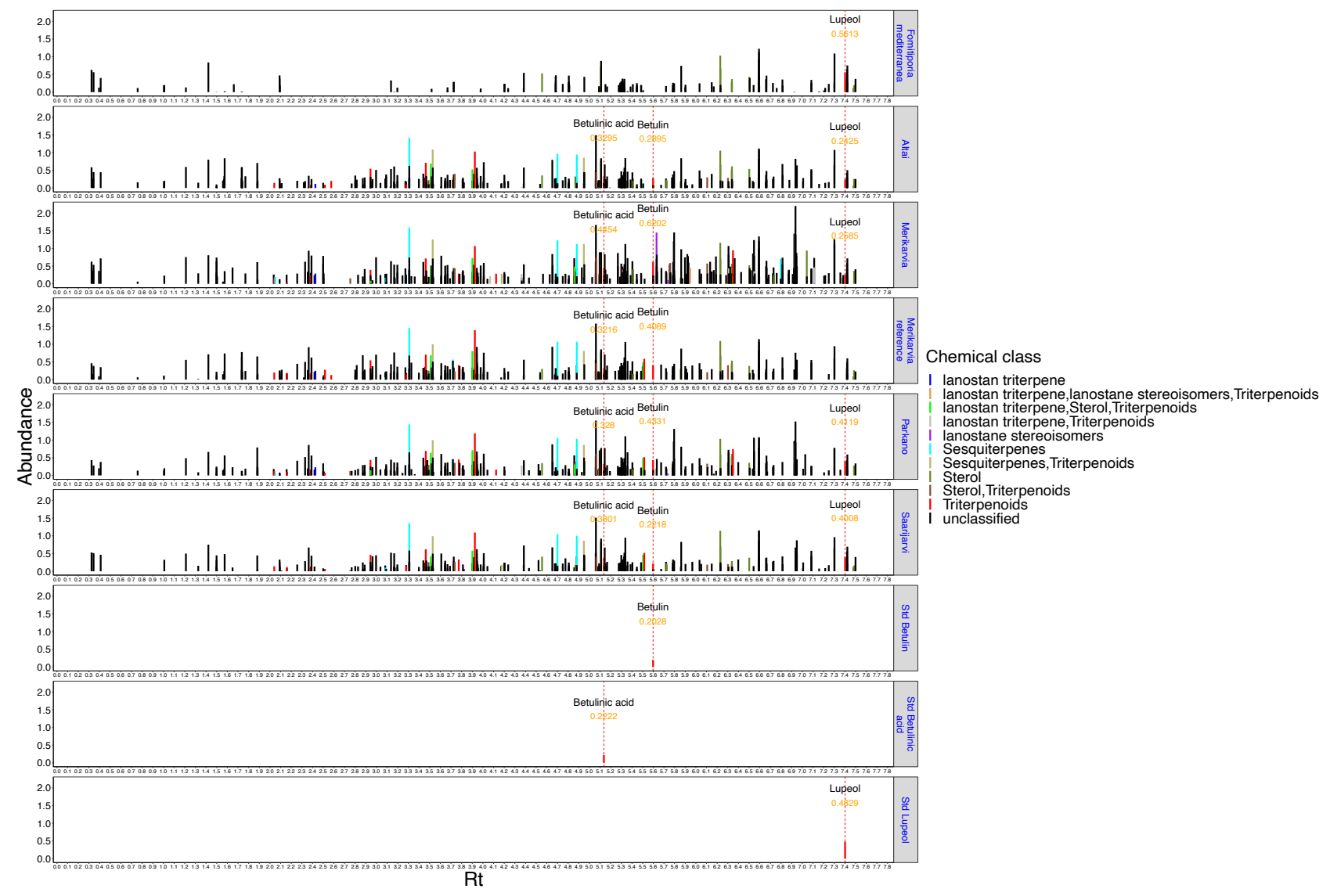

Supp. Fig 2: Relative abundances of secondary metabolites among five strains of $I$. obliquus, one strain of $F$. mediterranea, and standard (98\%) lupeol, betulin, and betulinic acid. $X$-axis is the retention time, and Y-axis the relative abundance (ggplot, scales='free_X'). Vertical red lines show the standard retention times for lupeol, betulin, and betulinic acid, labeled with the name of the compound and its relative abundance. Mass spectra are classified to known chemical class. Std: standard (98\%). 
A

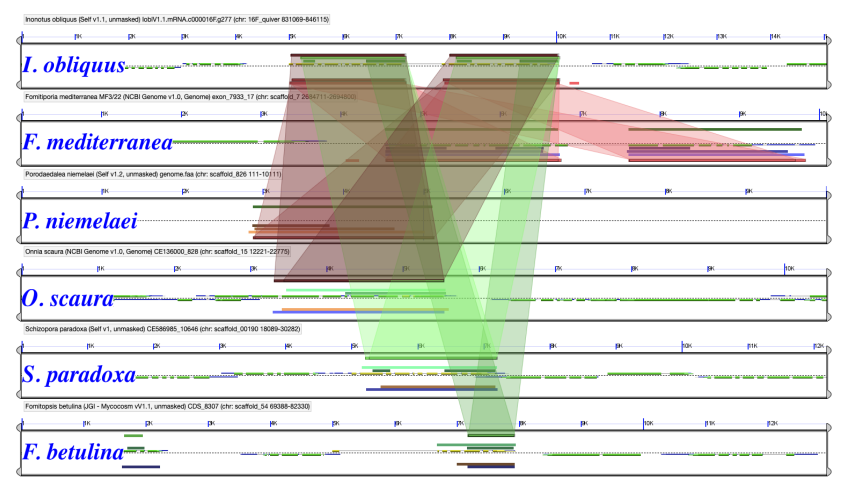

$\mathrm{C}$

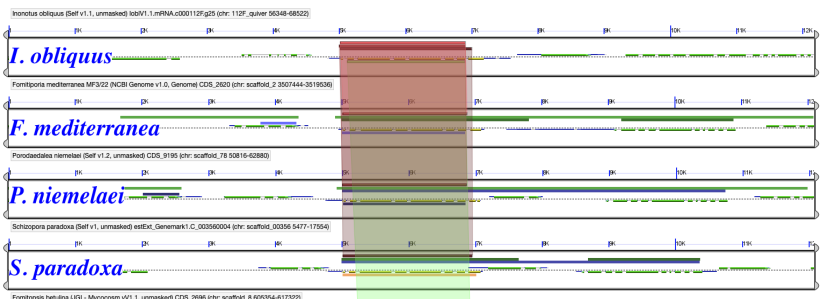

B

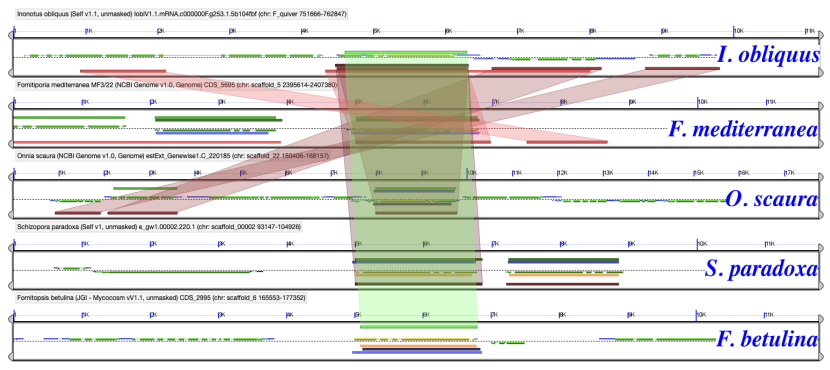

$\mathrm{D}$

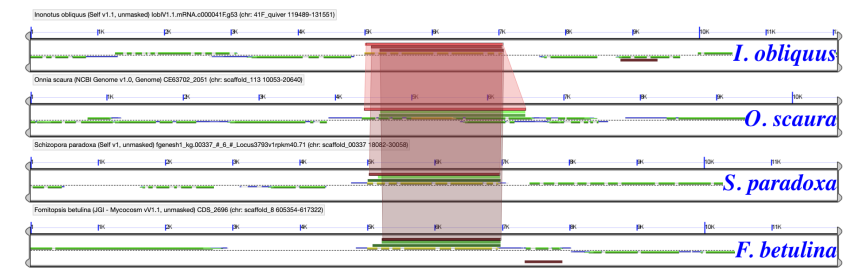

Supp. Fig 3: Microsynteny analysis of four cloned genes from I. obliquus and four Hymenochaetales species and Fomitopsis betulina. A) gene ID : c000016F.g277, B) gene ID : c000000F.g253, C) gene ID : c000112F.g25, and D) gene ID : c000041F.g53. 
Supp. table 1: Statistics of I obliquus genome assembly and annotation, genome size of orthologous species, repeat masking statistics, and the GO enrichment of genes adjacent to DNA transposable elements.

Supp. table 2: List of putative secreted proteins from I obliquus.

Supp. table 3: List of genes from I obliquus which are associated to syntenic or tandemly duplicated regions of the genome.

Supp. table 4: List of expanded gene families and their GO enrichment in I. obliquus.

Supp. table 5: List of homologous CAZymes from I. obliquus.

Supp. table 6: List of syntenic and tandemly duplicated genes, and gene ontology (GO) enrichment in these genomic regions.

Supp. table 7: List of metabolomics fingerprints from five strains of $I$. obliquus, and one strain of $F$. mediterranea.

Supp. table 8: List of putative oxygen, heme, ERR-Triad domains across multiple kingdoms.

Supp. table 9: List of differentially expressed gene families. 